\title{
The length of stellar bars in SB galaxies and $\mathbf{N}$-body simulations
}

\author{
L. Michel-Dansac ${ }^{1}$ and H. Wozniak ${ }^{2}$ \\ 1 Instituto de Astronomía, UNAM, Apartado Postal 877, 22800 Ensenada, BC, México \\ e-mail: leo@astrosen.unam.mx \\ 2 Centre de Recherche Astronomique de Lyon, 9 avenue Charles André, 69561 Saint-Genis Laval Cedex, France
}

Received 6 April 2004 / Accepted 14 December 2005

\begin{abstract}
Aims. We have investigated the accuracy and reliability of six methods used to determine the length of stellar bars in galaxies or $N$-body simulations.

Methods. All these methods use ellipse fitting and Fourier decomposition of the surface brightness. We have applied them to $N$-body simulations that include stars, gas, star formation, and feedback. Stellar particles were photometrically calibrated to make $B$ and $K$-band mock images. Dust absorption is also included. We discuss the advantages and drawbacks of each method, the effects of projection and resolution, as well as the uncertainties introduced by the presence of dust.

Results. The use of $N$-body simulations allows us to compare the location of Ultra Harmonic Resonance (UHR or 4/1) and corotation (CR) with measured bar lengths. We show that the minimum of ellipticity located just outside the bulk of the bar is correlated with the corotation, whereas the location of the UHR can be approximated using the phase of the fitted ellipses or the phase of the $m=2$ Fourier development of the surface brightness. We give evidence that the classification of slow/fast bars, based on the ratio $\mathcal{R}=R_{\mathrm{CR}} / R_{\mathrm{bar}}$ could increase from 1 (fast bar) to 1.4 (slow bar) just by a change of method. We thus conclude that one has to select the right bar-length estimator depending on the application, since these various estimators do not define the same physical area.
\end{abstract}

Key words. galaxies: evolution - galaxies: kinematics and dynamics - galaxies: spiral - galaxies: structure methods: $N$-body simulations

\section{Introduction}

Bars are ubiquitous and their importance for the long-term evolution of galactic discs is well established nowadays (e.g. see reviews by Sellwood \& Wilkinson 1993; Kormendy \& Kennicutt 2004, and references therein). One of the long-standing problems remaining for stellar bars is how to observationally delimit the area of a bar, i.e. to find where a bar morphologically ends or, in other words, how to measure its shape, length, and/or its width. Indeed, determining many crucial observational quantities relies on the definition of the bar size. However, a bar is not just an add-on morphological structure embedded into an axisymmetric disc+bulge background. In fact, some bulges (or pseudo-bulges, Kormendy \& Kennicutt 2004) could be the result of the secular evolution of bars, whereas part of the bar's stellar material spends a significant fraction of time far out into the disc (the so-called "hot" population, Sparke \& Sellwood 1987; Pfenniger \& Friedli 1991). This is the main reason any attempt to decompose $1 \mathrm{D}$ photometric profiles or 2D surface brightness in a number of elementary contributions (bulge(s), disc(s), etc.) to recover the "real" bar by substraction, in most cases, is doomed to failure.

However, one of the simplest quantities used to estimate the strength of a bar is its ellipticity. For instance, Martin (1995) found a correlation between the bar-axis ratio measured on blue photographic plates and star-formation activity in a sample of 136 barred galaxies. Chapelon et al. (1999) confirm this correlation using a more reliable photometry on red CCD images. Using a sub-sample of Martin's data, Martinet \& Friedli (1997) emphasize that the most active galaxies have the longer and thinner bars, but they also conclude that this is only a necessary condition, not a sufficient one. However Knapen et al. (2002) argue that this result could be dependent on the techniques used to determine bar lengths and strengths. Moreover, the bar strength estimator $Q_{\mathrm{b}}$ depends both on the axis ratio and the mass of the bar (Sanders \& Tubbs 1980).

There is another set of studies that needs accurate bar-length measurements. Numerous authors have tried to correlate the size of morphological structures to dynamical resonance locations. Circumnuclear and outer rings seem to be correlated with the location of, respectively, the inner Lindblad resonance (ILR) and the outer Lindblad resonance (OLR) (cf. Buta \& Combes 1996). The ratio of the nuclear bar length to the large-scale bar length could be similar to the ILR-to-corotation ratio, the nuclear bar corotation being dynamically coupled with the large-scale bar ILR (e.g. Rautiainen \& Salo 1999). However, some other simulations did not show such coupling (e.g. Heller et al. 2001). In short, this matter is still being debated.

The extent of the bar (either nuclear or large-scale) should thus be compared to the corotation location. There are several observational evidences (Kent 1990 and references therein) that stellar bars must end before the corotation. On the theoretical side, the theory of orbits (Contopoulos 1980), hydrodynamic simulations (Sanders \& Tubbs 1980; Athanassoula 1992b; Regan \& Teuben 2004; etc.), and $N$-body simulations (Sparke \& Sellwood 1987) all agree to predict that the ratio of the corotation radius to the bar length is $1.2 \pm 0.2$. Physically, this limitation is due to an increase in the amount of chaotic orbits close to the corotation. Thus, near the corotation, there is no orbital support to prolongate the shape of the bar. However, it is difficult to observationally confirm this value since it not only depends on the method used to determine the pattern speed, but also on the 
criterion used to determine the bar length. For instance, Aguerri et al. (2003) used four different criteria to determine the bar length. Recent studies of the interaction between a stellar disc and a live dark halo have widely discussed the evolution of the ratio of the corotation radius over the bar length, implicitly outlining the importance of an accurate bar-length determination (Debattista \& Sellwood 2000; Athanassoula \& Misiriotis 2002; Athanassoula 2003; O’Neill \& Dubinski 2003; Valenzuela \& Klypin 2003).

Another difficulty arises from the morphological difference between mass and multi-wavelength surface-brightness distributions. Indeed, with $N$-body simulations, the bar length is determined on the mass distribution, whereas observational determinations use wavelength dependent surface-brightness distributions. Comparisons between the two imply an understanding and an estimation of the no-linear bias between mass and light. It poses, among others, the question of the reliability of the various criteria used in the literature and what they physically determine, depending on the support (mass or light) on which they are applied.

To test the efficiency, reliability, and accuracy of various criteria for determining the length of a bar, we decided to apply them to $N$-body simulations that include stars, gas, and starformation recipes. These simulations were photometrically calibrated, taking dust extinction into account (cf. Michel-Dansac \& Wozniak 2004, hereafter Paper I). Thus, we could perform a systematic comparison of methods applied to mass distributions, as well as to photometrically calibrated mock images in blue and near-infrared wavelengths. Then, the location of the bar ends, estimated using six different techniques, was compared to the location of dynamical resonances. We thus exploit the comprehensive knowledge of the dynamical properties of the simulation to find the best bar-length estimator.

In Sect. 2 we describe the six criteria used to measure bar lengths. We also discuss some of their relative advantages and drawbacks. Then, in Sect. 3, we describe our numerical models and their evolution. We also briefly recall the technique of photometric calibration used in Paper I. The six criteria are applied to our simulations in Sect. 4. We also analyse inclination effects on our results in this section. Section 5 deals with the temporal evolution of bar lengths. Correlations between the various bar length determinations and the location of dynamical resonances are studied in Sect. 6. The distinction between fast and slow bars and the limitations due to the lack of dark halo in our simulations are discussed in the same section. We finally summarise our results in the last section.

\section{Measurements of bar length}

The method adopted by Martin (1995) for measuring the length and width of bars is exclusively visual and relies on photographic prints in the blue band. He estimates that the uncertainty is about $20 \%$ with this method. He defines the half-major axis of the bar as the length from the galaxy centre to the sharp outer tip where spiral arms begin, and the half-minor axis as the length from the centre to the edge of the bar, oval, lens, or spheroidal component, measured perpendicularly to the major axis. Using CCD red images, Chapelon et al. (1999) made a more sophisticated numerical analysis. They first extracted a 3-pixel wide photometric profile along the major axis of the bar. The half-major axis of the bar is the distance from the centre of the galaxy to where the bar obviously ends. This is where the surface-brightness profile changes slope abruptly to become steeper; this also coincides with the origin of the spiral arms.
This measurement is not automatic, as it relies on a subjective judgement of where the bar ends, and is comparable to that of Martin (1995).

Beyond eye estimates, several attempts to find an automatic and objective criterion were made to determine where the bar ends and the disc or the spiral arms start. The objective criteria can be classified in two groups defined by the tools that used: 3 based on ellipse fitting (noted $E_{1}$ to $E_{3}$ ), and 3 based on the Fourier analysis (noted $F_{1}$ to $F_{3}$ ). We applied all six criteria on the mass distribution and on the calibrated images.

From now on, we use the expression "bar radius" instead of "bar length" since the various criteria used in this paper deal with either half-major axis length or radius. Each measurement must obviously be doubled whenever the real "length" or the "diameter" should be determined.

\subsection{Ellipses fitting}

In the past, the analysis of isophotal shapes using a functional form was done using either classical ellipses (e.g. Wozniak \& Pierce 1991; Wozniak et al. 1995) or generalised ellipses (Athanassoula et al. 1990), which permits the description of boxy-like isophotes.

By fitting ellipses to the isophotes of a large sample of barred galaxies, Wozniak et al. (1995) determined some general rules about the radial behaviour of both the ellipticity (defined by $e=1-b / a$ where $a$ and $b$ are the half-major and half-minor axis lengths respectively) and the position-angle (PA) of the isophotes. The ellipticity is minimum or vanishes at the centre, because of either seeing effects or a luminous spherical bulge; then it increases to reach a maximum $e^{\max }$, often at about the middle of the bar, and then progressively decreases towards $e^{\min }$ at the place where the isophotes should become axisymmetric (disc) in the face-on case. Of course, $e^{\min }$ is determined by the galaxy inclination and/or the real non-axisymmetric shape of the disc. The PA is constant along the bar and, in general, sharply takes another constant value for the disc isophotes. If a spherical bulge is present, $e$ will only start to increase outside the bulge.

Three criteria can be defined using the results of ellipse fitting. For the first criterion $\left(\mathrm{E}_{1}\right)$, the bar radius is the radius where the ellipticity profile reaches a maximum. It has been introduced by Wozniak \& Pierce (1991), who studied a sample of SB0 galaxies in optical wavebands. Although this criterion is very useful for automated measurements (e.g. Regan \& Elmegreen 1997; Jungwiert et al. 1997; Laine et al. 2002) because the maximum ellipticity is always clearly defined in SBO galaxies, it seems to give rather short values of bar radius when it is compared to eye estimates (cf. also Fig. 7).

Wozniak et al. (1995) later introduced another criterion (hereafter called $\mathrm{E}_{3}$ ) given by the position where the PA variation exceeds $5^{\circ}$ and ellipticity drops to disc values (rounder isophotes). This criterion gives higher values that were expected to be upper limits of real bar radii. Erwin \& Sparke (2003) used a PA variation of $10^{\circ}$ instead of 5 , which should lead to slightly longer bars. The regions where the $E_{3}$ criterion is satisfied are generally less dusty than for $E_{1}$ since gas density strongly decreases outside the bar. The bar-radius estimation using $\mathrm{E}_{3}$ might be more robust for most galaxies than $E_{1}$ with respect to extinction but, depending on the detailed spatial distribution of the gas, could also lead to large errors. A variant of the $E_{3}$ criterion has been used by Jogee et al. (2004) to analyse almost 1500 barred galaxies in the ACS GEMS survey, showing its reliability in data-mining studies. 


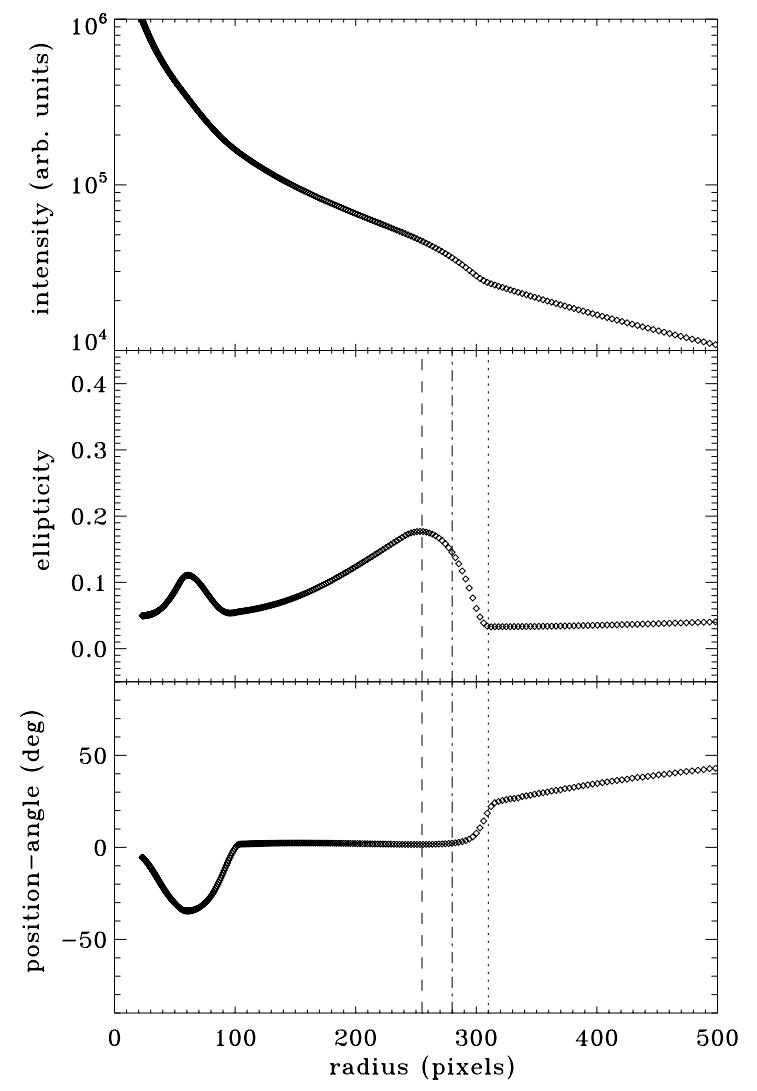

Fig. 1. Illustrative example of the profiles obtained by ellipse fitting. From top to bottom: surface brightness in arbitrary units, ellipticity, and PA (in degrees) profiles as a function of half-major axis of the fitted ellipse. Each point represents a fitted ellipse. The vertical lines represent bar radii determined with $\mathrm{E}_{1}$ (dashed line), $\mathrm{E}_{2}$ (dot-dashed line), and $\mathrm{E}_{3}$ (dotted line).

We now define a new criterion $\left(E_{2}\right)$ that is also based on ellipse fitting. In the region of the bar, where the ellipticity profile looks like a bump, the PA is approximately constant, hence the PA profile forms a plateau (cf. Fig. 1). For the $E_{2}$ criterion, bar radius is measured at the end of this plateau. This corresponds to a twist between isophotes of the bar and isophotes of the disc region where spiral arms begin. This criterion gives an estimation of bar radii between those of $E_{1}$ and $E_{3}$ criteria in the absence of dust extinction.

To illustrate how these criteria could lead to different barradius estimations, we made an artificial image that contains a bulge, two embedded bars, and a disc. Our purpose is not to make an extensive study, varying each parameter to quantify the quality of each bar-radius estimator. The bulge has a de Vaucouleurs profile of central intensity $I_{\mathrm{e}}=9000$, scale length $R_{\mathrm{e}}=200$. It is slightly elongated along $\mathrm{PA}=0^{\circ}$ with an ellipticity of 0.05 . The exponential disc has a central intensity $I_{\mathrm{d}}=8000$, scale length $R_{\mathrm{d}}=300$, and an ellipticity of 0.1 . The PA of the disc's main axis is PA $=60^{\circ}$. The two bars have a surface density:

$I(x, y)=I_{i}\left(1-\left|\frac{x}{a_{i}}\right|^{c_{i}}-\left|\frac{y}{b_{i}}\right|^{c_{i}}\right)$

where $a_{i}$ is the half-major axis (i.e. bar radius), $b_{i}$ the halfminor axis, $I_{i}$ the central intensity, and $c_{i}$ the shape parameter (Athanassoula et al. 1990). The main bar has $a_{1}=300, b_{1}=200$, $I_{1}=5000$, and $c_{1}$ varies from 2 at the centre (perfect ellipses) to 3.5 at the ends, thus giving a rectangular-like bar. It is aligned with the $x$-axis. A nuclear bar is added at $\mathrm{PA}=-45^{\circ}$ with the same intensity profile with parameters $a_{2}=100, b_{2}=50, c_{2}=2$ everywhere, and $I_{2}=16000$. All scale units are pixels and intensity units are numbers that could be considered as ADU.

We display the results of the ellipse fitting in Fig. 1, i.e. the ellipticity and PA of the isophotes as a function of the half-major axis length of the fitted ellipse. Applying the above criteria leads to a bar radius of 255 for $E_{1}, 280$ for $E_{2}$, and 310 for $E_{3}$. This last criterion slightly overestimates the real bar radius because of the rectangular shape of the isophotes near the end of the bar. Ellipses are indeed not suited to strong rectangular bars.

\subsection{Fourier analysis}

Fourier analysis of the surface brightness (Ohta et al. 1990) is based on the decomposition of azimuthal density profiles, $I(r, \theta)$, into a Fourier series

$I(r, \theta)=I_{0}(r)+\sum_{m=1}^{\infty}\left[A_{m}(r) \cos (m \theta)+B_{m}(r) \sin (m \theta)\right]$

where, for $m \neq 0$, the coefficients are given by:

$A_{m}=\frac{1}{\pi} \int_{0}^{2 \pi} I(r, \theta) \cos (m \theta) \mathrm{d} \theta$
$B_{m}=\frac{1}{\pi} \int_{0}^{2 \pi} I(r, \theta) \sin (m \theta) \mathrm{d} \theta$.

The Fourier amplitude of the $m$ th component $(m>0)$ is defined as:

$I_{m}(r)=\sqrt{A_{m}^{2}(r)+B_{m}^{2}(r)}$.

The bar-interbar contrast is then computed as:

$C=I_{\mathrm{b}} / I_{\mathrm{ib}}=\left(I_{0}+I_{2}+I_{4}+I_{6}\right) /\left(I_{0}-I_{2}+I_{4}-I_{6}\right)$.

We display $C$ in Fig. 2 for the test image used above. Typically, $C$ increases steeply and reaches its peak value in the bar region, then falls toward the bar end. The bar region is defined by Ohta et al. (1990) as the zone where the contrast exceeds $2\left(F_{1}\right.$ criterion hereafter). This criterion has been revisited by Aguerri et al. (2000), who redefine the end of the bar region where the contrast reaches the full width at half maximum (criterion $\mathrm{F}_{2}$ ). In other words, the bar ends where $C=0.5[\max (C)+\min (C)]$.

Another criterion $\left(\mathrm{F}_{3}\right)$ is determined using the radial profile of the phase of the $m=2$ component, which is defined as:

$\phi_{2}(r)=\arctan \left[B_{2}(r) / A_{2}(r)\right]$.

We display this phase profile in Fig. 2 for the test image. The $m=2$ phase can be interpreted as the PA of the features that dominate the $m=2$ component. Thus, the phase successively gives the PA of the bar, then of the two-arm spiral structure, if present, or/and the PA of the disc (if not perfectly disky, which is always the case, due to inclination at least). The $m=2$ phase profile is then similar to the PA profile, and the phase in the bar region is approximatively constant. We measure bar radius at the end of this plateau. This criterion has already been applied to mass distribution from $N$-body simulations (e.g. Debattista \& Sellwood 2000), as well as to observations (e.g. Aguerri et al. 2003).

These criteria applied to the artificial image lead to a bar radius of $\approx 280$ for $F_{2}$, whereas $F_{3}$ gives $\approx 270$. However, $F_{1}$ fails to give any estimation, since the contrast never exceeds the value of 2 . Thus, for the case of our test image, $F_{2}$ and $F_{3}$ underestimate the radius of the main bar. 
Table 1. Initial parameters of the simulations.

\begin{tabular}{lllllllllll}
\hline \hline Run & $N_{\mathrm{s}}$ & $N_{\mathrm{g}}$ & $M_{1}$ & $M_{2}$ & $M_{\mathrm{g}}$ & $a_{1}$ & $a_{2}$ & $b$ & $a_{\mathrm{g}}$ & $b_{\mathrm{g}}$ \\
& & & & & & & & & & \\
\hline A & 500000 & 50000 & $10^{10}$ & $10^{11}$ & $1.1 \times 10^{10}$ & 0.5 & 3.0 & 0.5 & 3.0 & $10^{-4}$ \\
B & 500000 & 10000 & $10^{10}$ & $10^{11}$ & $3.66 \times 10^{10}$ & 0.5 & 3.0 & 0.5 & 3.0 & 0.5 \\
C & 600000 & 10000 & $2 \times 10^{10}$ & $10^{11}$ & $4 \times 10^{10}$ & 0.5 & 5.0 & 1.0 & 10.0 & 0.5 \\
\hline
\end{tabular}

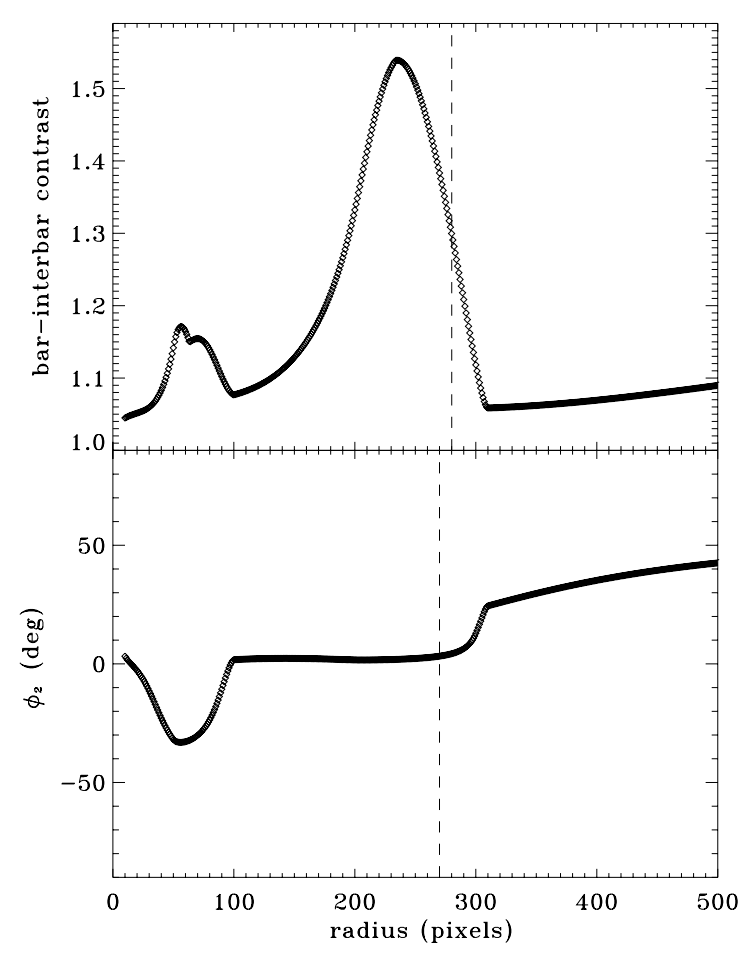

Fig. 2. Illustrative example of the profiles obtained by Fourier analysis: bar-interbar contrast (top) and phase of the $m=2$ component (bottom) as a function of radius. Each point represents a pixel. The dashed vertical lines symbolise the end of the bar determined using $F_{2}$ (top) and $F_{3}$ (bottom) criteria.

\section{Numerical models}

\section{1. hydro+N-body simulations}

We used PMSPHSF, the $N$-body code developed in Geneva. It includes stars, gas, and recipes to simulate star formation. The broad outlines of the code are as follows: the gravitational forces are computed with a particle-mesh method using a 3D polar grid (Pfenniger \& Friedli 1993), the hydrodynamics equations are solved using the smooth particle hydrodynamics (SPH) technique (see Friedli \& Benz 1993, for this implementation; and Monaghan 1992 for a review of the method), and the starformation process is based on Toomre's criterion for the radial instability of gaseous discs (Friedli \& Benz 1995). For the present work, the radiative cooling of the gas was computed assuming a solar metallicity for Runs A and B and a cosmological metallicity for Run C. The metallicity change of gas particles was computed assuming net yields given by Maeder (1992). At birth, star particles are created from gas, so they have the same metallicity.

An initial stellar population was set up to reproduce a disc galaxy with an already formed bulge. These particles form what we call hereafter the "initial population", as opposed to particles created during the evolution ("new population"). The initial stellar positions and velocities for $N_{\mathrm{s}}$ particles of the same mass

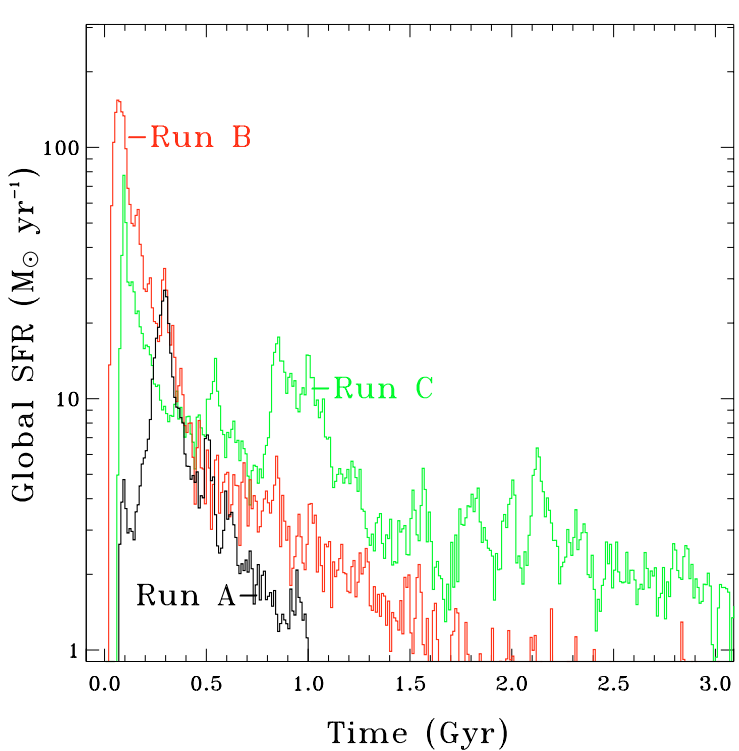

Fig. 3. Evolution of the star-formation rate (SFR) of Runs A (black), B (red), and C (green).

are drawn from a superposition of two axisymmetric MiyamotoNagai discs of mass $M_{1}$ and $M_{2}$, of scale lengths $a_{1}+b$ and $a_{2}+b$, respectively, and identical scale height $b$ (cf. Table 1, where masses are in $M_{\odot}$ and lengths in kpc). Their gravitational potential is then given by (Miyamoto \& Nagai 1975):

$$
\Phi_{1,2}(R, z)=-\frac{G M_{1,2}}{\sqrt{R^{2}+\left(a_{1,2}+\sqrt{z^{2}+b^{2}}\right)^{2}}}
$$

where $R=\sqrt{x^{2}+y^{2}}$. The gaseous component is represented by $N_{\mathrm{g}}$ particles for a total mass $M_{\mathrm{g}}$, scale length $a_{\mathrm{g}}+b_{\mathrm{g}}$, and scale height $b_{\mathrm{g}}$. As in Paper I, we did not include a dark halo, to be able in the near future to compare the effects of having added such an additional component.

Three simulations were used. Run A (cf. Fig. 4) was computed over 1 Gyr. It was analysed in some detail in Paper I. Runs B (Fig. 5) and C (Fig. 6) were performed on a longer timescale. They will be used to check our results on the long term with different initial conditions. The main differences between the three simulations are the initial mass of gas and its spatial distribution. The resulting star-formation histories are quite different (cf. Fig. 3): Run A is more representative of a short timescale burst of star formation; Run $C$ shows several small events but star formation is sustained over a longer period because of the more massive gaseous reservoir initially distributed over a greater length scale than for Run A; Run B is an intermediate case.

During the three simulations, a bar progressively grows and then evolves secularly. The gravitational torques due to the bar and spiral structures drive the gas inwards. Star formation occurs along the transient spiral structure, along and around the bar, and in the central parts. The bar formation is thus associated to the 

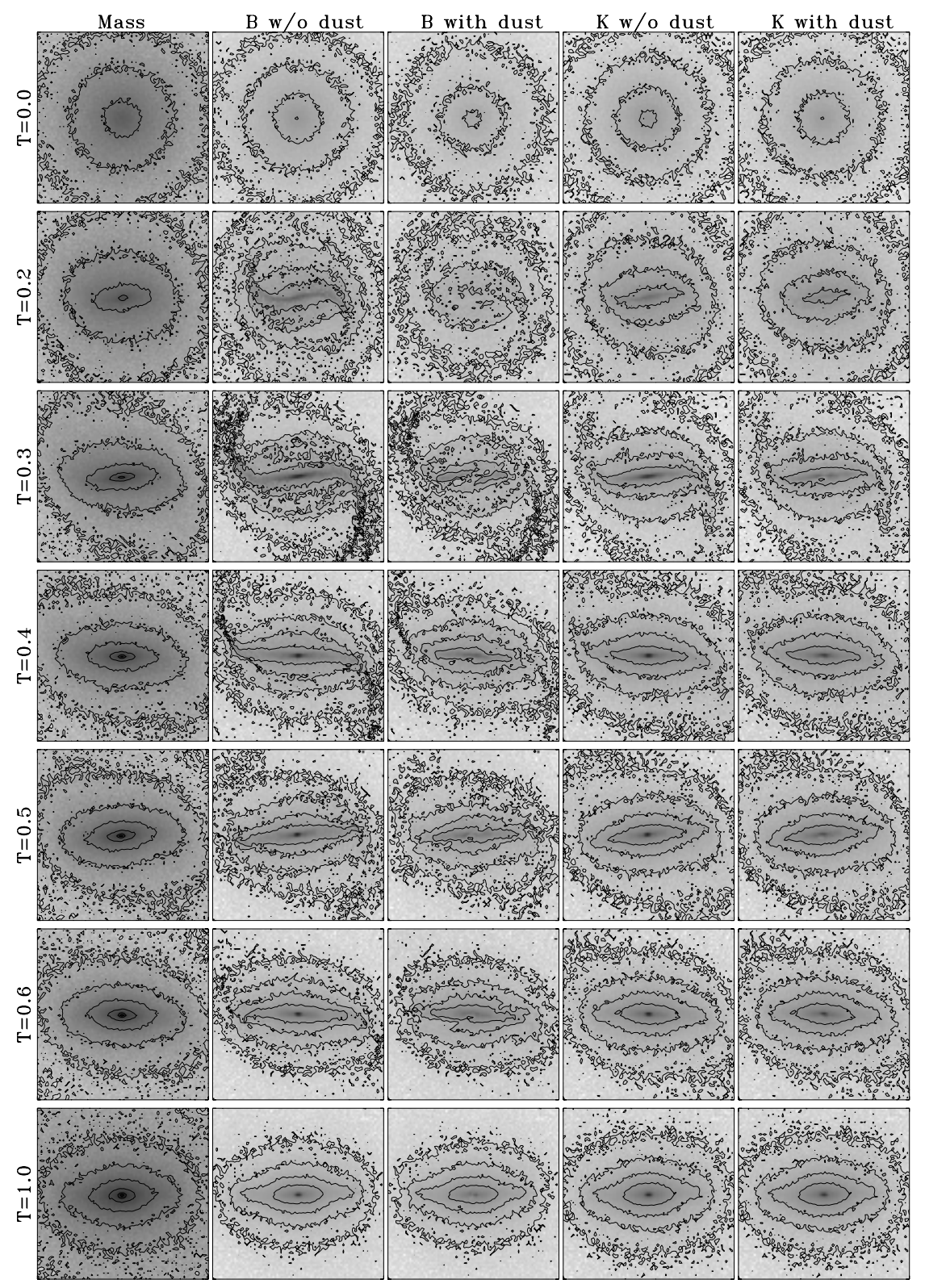

Fig. 4. Evolution of Run A from $t=0$ to $t=1$ Gyr. From left to right are displayed the mass distribution (in $\log$ of $M_{\odot} \mathrm{pc}^{-2}$ ) and the calibrated images (B dust-free, B with extinction, $\mathrm{K}$ dust-free, and $\mathrm{K}$ with extinction) in mag $\operatorname{arcsec}^{-2}$. The field of view (10 kpc) is the same for each frame. The particles have been rotated so that the bar position is roughly horizontal. Greyscale images and isocontours of mass surface density range from $10^{1}$ to $10^{5.2} M_{\odot} \mathrm{pc}^{-2}$. Mass isocontours are logarithmically spaced by $0.47 \log \left(M_{\odot} \mathrm{pc}^{-2}\right)$. The $B$ greyscale images range from 11 to $26 \mathrm{mag} \operatorname{arcsec}^{-2}$, while contours range from 20 to $23 \mathrm{mag} \mathrm{arcsec}^{-2}$ and are spaced by 1 mag. The $K$ images range from 9 to $22 \mathrm{mag} \operatorname{arcsec}{ }^{-2}$; $K$ isocontours range from 17 to $20 \mathrm{mag} \operatorname{arcsec}^{-2}$ and are spaced by 1 mag.

starburst seen in Fig. 3. The bar-formation timescale differs from one simulation to the next, as a consequence of the different initial conditions. Another consequence is the morphological difference in the resulting bars. With these simulations, we have thus obtained a collection or "sample" of snapshots with a wide variety of bar shape, as can be seen in Figs. 4-6; note the larger field of view used in Fig. 6 than in the other two. Moreover, our simulated bars have properties (such as ellipticity or strength) representative of nearby galaxies, as discussed in Sect. 4.

\subsection{Mock calibrated images}

Dynamical properties of a bar obviously depend on the mass distribution. Collisionless $\mathrm{N}$-body simulations are thus one of the best tools for studying them. However, when one deals with determining observational properties (i.e. photometric properties in our case), an additional dependency makes the use of collisionless $N$-body simulations much more difficult: the mass-to-light ratio $(M / L)$. Indeed, $M / L$ varies in time and space since it depends both on the star-formation history and on the mixing of stellar populations by secular evolution. Simulations with gas and star formation permit photometrically calibrated light distributions to be obtained by performing a post-processing calibration with stellar-population synthesis models, as described hereafter.

For each stellar particle, given its age and metallicity, the mass-to-light ratio was obtained from a bi-linear interpolation of the tables of GISSEL2000 (Bruzual \& Charlot 1993), for a Salpeter initial mass function with a lower mass cutoff $M_{\mathrm{d}}=$ $0.1 M_{\odot}$ and a upper cutoff $M_{\mathrm{u}}=100 M_{\odot}$. 

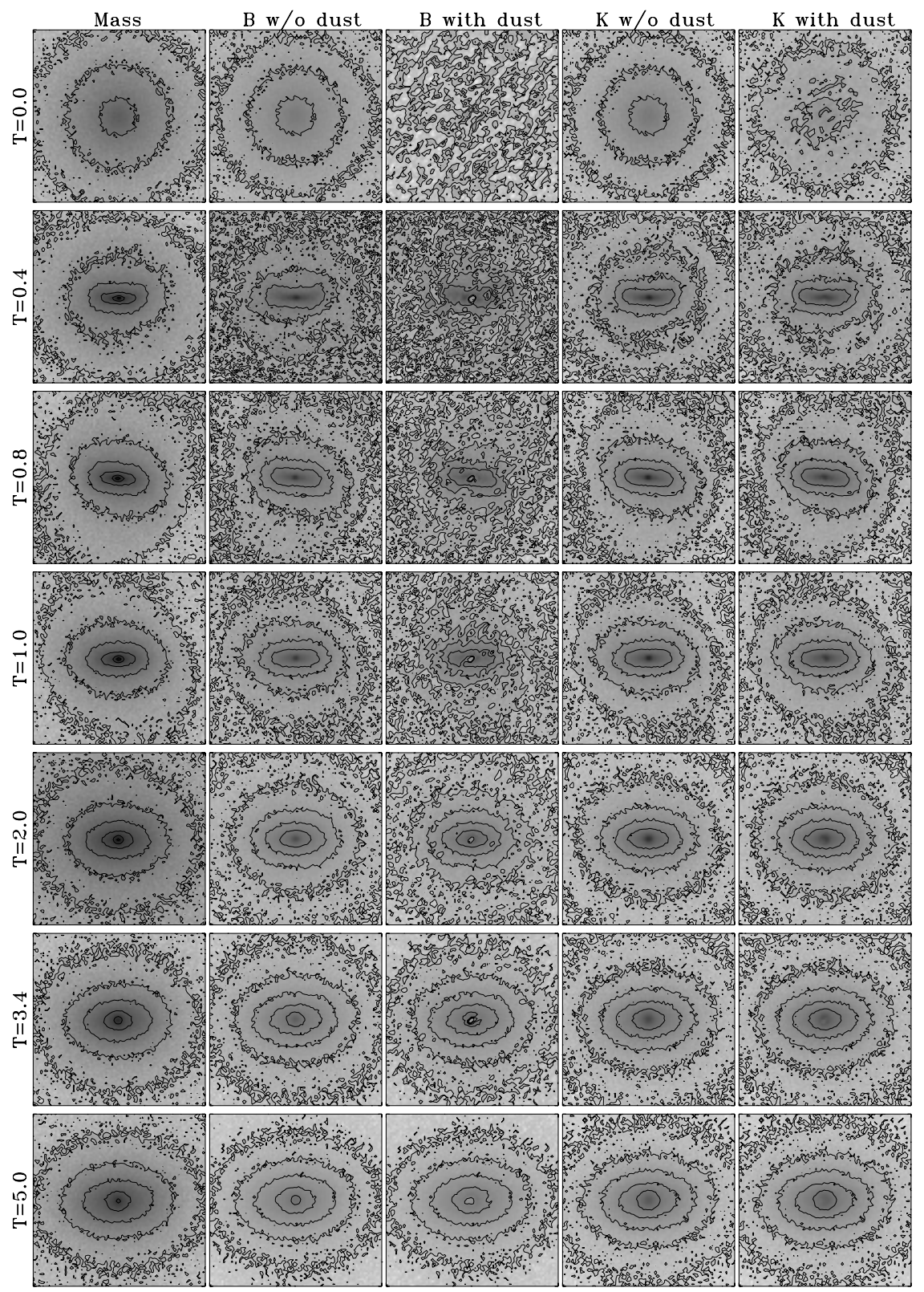

Fig. 5. Like Fig. 4 but for Run B from $t=0$ to $t=5 \mathrm{Gyr}$. Greyscale images and isocontours of mass surface density range from $10^{1}$ to $10^{5.2} M_{\odot} \mathrm{pc}^{-2}$. Mass isocontours are logarithmically spaced by $0.47 \log \left(M_{\odot} \mathrm{pc}^{-2}\right)$. The $B$ greyscale images range from 12 to $27 \mathrm{mag}^{\text {arcsec }}{ }^{-2}$, while contours range from 19 to $23 \mathrm{mag} \operatorname{arcsec}^{-2}$ and are spaced by $1 \mathrm{mag}$. The $K$ images range from 10 to $23 \mathrm{mag} \operatorname{arcsec}^{-2} ; K$ isocontours range from 16 to $20 \mathrm{mag} \operatorname{arcsec}^{-2}$ and are spaced by 1 mag.

For the initial population of Run A (present at the beginning of the simulation), we assumed an age of $10.4 \mathrm{Gyr}$ and metallicity of $Z=0.004$, like the model A of Paper I. In that paper, we considered various calibrations of the initial population and found that the length of the bar (determined with the $\mathrm{E}_{3}$ criterion, defined above) is almost independent of the calibration model. We thus decided here to restrict our study to one calibration model for the sake of clarity.

Obviously, the assumptions on the age and metallicity of the initial population implicitly imply that all particles were simultaneously born $10.4 \mathrm{Gyr}$ before the beginning of the simulation. Assuming an observer located at $z=0$ at the end of the simulation $(t=1 \mathrm{Gyr})$, this calibration implies a redshift of formation of $z=3$. For the longer Runs B and C, we considered several sets of initial population ages and metallicities. For clarity, we deal only with the calibration model that assumes an initial population age of $4.4 \mathrm{Gyr}$ and a metallicity of $Z=10^{-4}$. This leads to a final age of $11.4 \mathrm{Gyr}$, the same as for Run A.

In Paper I we worked with four wavebands to cover the wavelength domain from the visible to the near infrared, namely $B, R, H$, and $K$ Johnson. Here, we restrict our analysis to $B$ and $K$ bands without any loss in generality.

Figures 4 to 6 display the evolution of the mass density and the $B$ and $K$ calibrated images with and without dust, in the bar region for a selection of snapshots. For Run A, large scale snapshots of the evolution can be found in Paper I. These figures clearly show that the disc undergoes a complete redistribution of the mass. For instance, for Run A after 1 Gyr, the total mass inside the central kpc has roughly doubled. The first cause of this mass inflow is the formation of a stellar bar. Then, due to the gravitational torques exerted on the gas by the stellar bar, the extra mass in the form of gas and new stars amounts to $3.5 \times 10^{9} M_{\odot}$ 


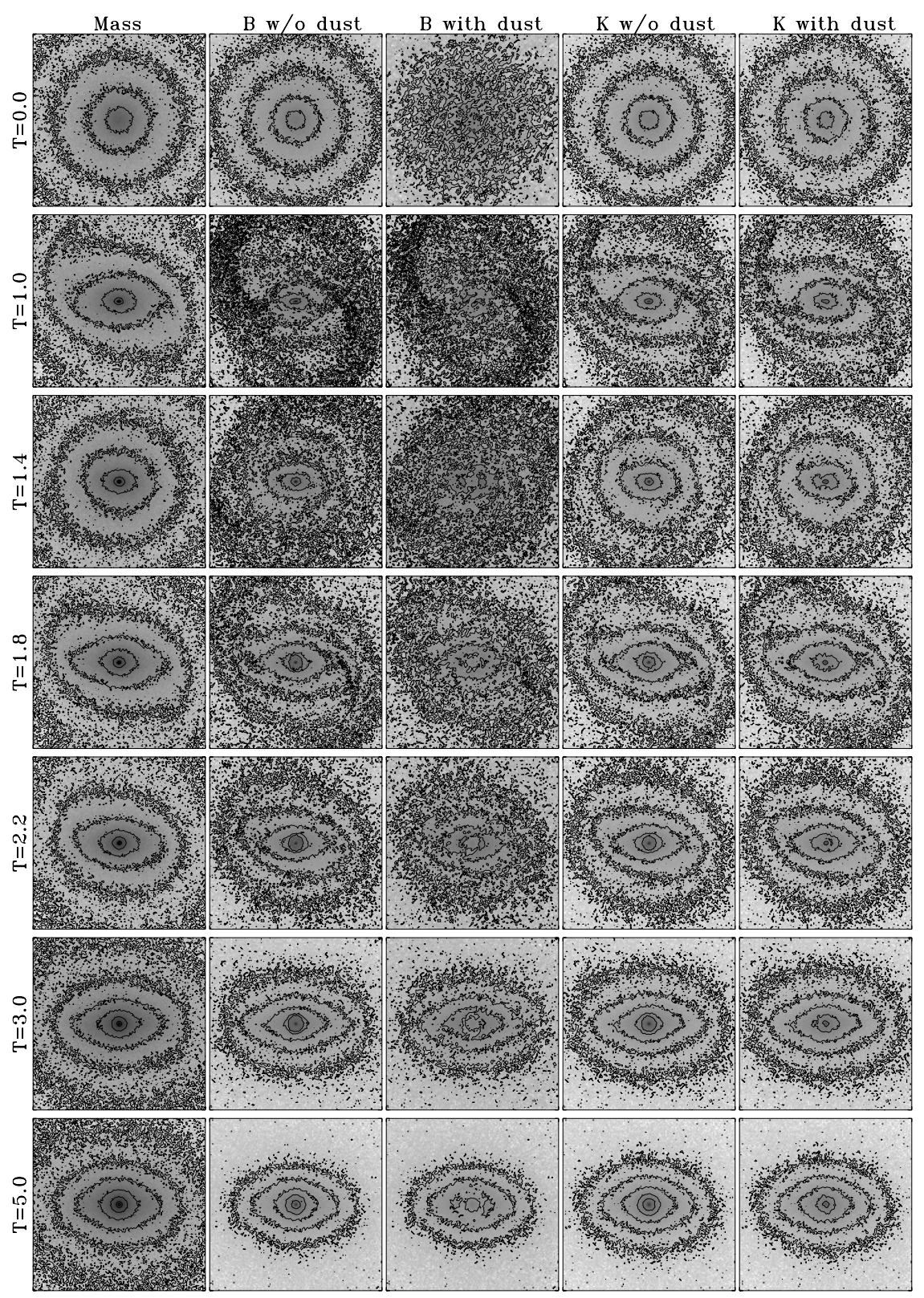

Fig. 6. Like Fig. 4 but for Run C from $t=0$ to $t=5 \mathrm{Gyr}$. The field of view is $16 \mathrm{kpc}$. Greyscale images and isocontours of mass surface density range from $10^{1}$ to $10^{5} M_{\odot} \mathrm{pc}^{-2}$. Mass isocontours are logarithmically spaced by $0.44 \log \left(M_{\odot} \mathrm{pc}^{-2}\right)$. The $B$ greyscale images range from 13 to $27 \mathrm{mag} \operatorname{arcsec}^{-2}$, while contours range from 19 to $23 \mathrm{mag} \mathrm{arcsec}^{-2}$ and are spaced by 1 mag. The $K$ images range from 10 to $23 \mathrm{mag}$ arcsec ${ }^{-2}$; $K$ isocontours range from 16 to $20 \mathrm{mag} \operatorname{arcsec}^{-2}$ and are spaced by 1 mag.

at $t=1$ Gyr for Run A, which is only $30 \%$ of the whole additional mass. In fact, the initial stellar population contributes to the other $70 \%$.

After individual particle photometric calibration, mock CCD images were obtained summing particle luminosities into a $512 \times$ 512 pixel grid. The field of view was $60 \mathrm{kpc}$, which gave a spatial resolution of $\approx 117 \mathrm{pc}, 1.3$ times our smallest $N$-body grid resolution. We thus produced one frame per waveband and per snapshot of the simulation. Our results are obviously independent of the bar PA with respect to the North or any other axis. We thus decided to systematically rotate the positions of particles to align the bar with the $x$-axis.

To mimic real observations we should have to convolve our images with a point-spread function. However, this last stage depends on the telescope and observation-site characteristics. It thus introduces a few free parameters that cannot be constrained without any detailed comparisons with real observations, which is not our purpose.

\subsection{Dust extinction}

Dust extinction in $B$ and $K$ bands was simulated assuming a constant gas-to-dust ratio

$N(\mathrm{H} \mathrm{I}) / A_{\mathrm{V}}=5.34 \times 10^{21} \mathrm{~cm}^{-2}$.

Then, $A_{\mathrm{V}}$ is converted to $A_{B}$ and $A_{K}$. Extinction was computed in a cube with the same spatial resolution as for our images and 11 slabs along the line-of-sight (cf. Paper I for more details). Each slab absorbs the stellar luminosity behind it. For each slab, the gas density distribution is obtained by convolving particle positions with the SPH kernel. 

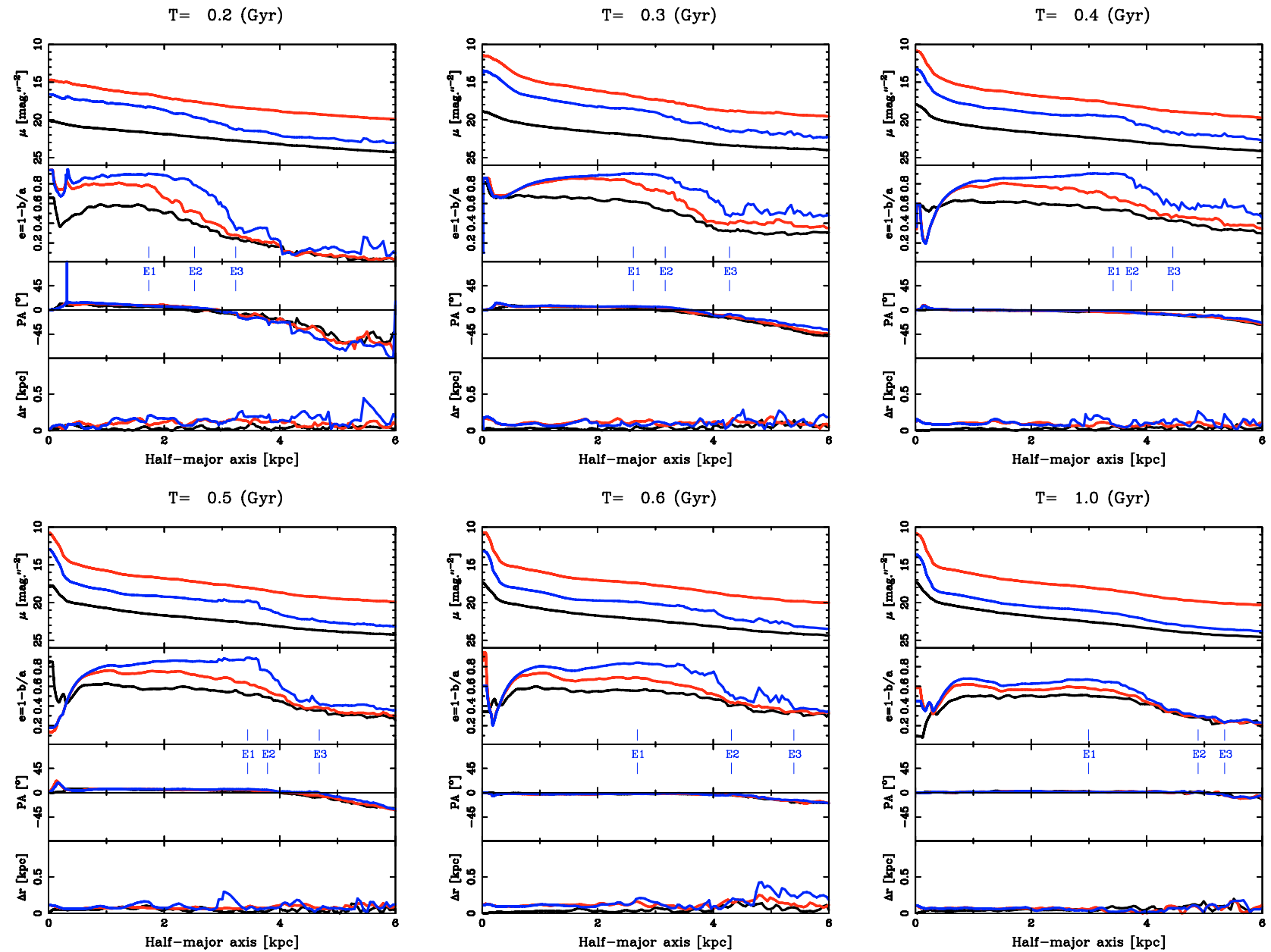

Fig. 7. In each panel, for the two bands ( $B$ plotted in blue and $K$ in red) and the mass distribution (in black) for Run A: from top to bottom, surface brightness $(\mu)$, ellipticity $(e=1-b / a)$, PA, and central shift for each fitted ellipse. The range in radius for the figures begins at $1 \mathrm{px}$, i.e. $117 \mathrm{pc}$; the upper bound corresponds to a region that encloses the bar during the entire run. The mass density profiles have been scaled so that the 30th magnitude corresponds to $1 M_{\odot} \mathrm{pc}^{-2}$. The labels $\mathrm{E}_{1}, \mathrm{E}_{2}$, and $\mathrm{E}_{3}$ refer to the criteria used to determine the bar radius in $B$ band. See the text for their definitions.

\section{Tests of bar-radii criteria on numerical simulations}

Each mock image was analysed by fitting simple ellipses to the isophotes. Profiles of surface brightness, ellipticities, and PA were obtained by increasing the half-major axis length $a$ by a factor 1.01 between each fit. We chose such a low value to obtain a good resolution in the inner region. We display the results for Run A in Figs. 7 and 8 for the same selected times as in Fig. 4. Results for the other runs are qualitatively similar, so they are not displayed.

The ellipticity profiles in both bands are significantly different for $t<1$ Gyr. In Paper I, we proposed that, even in the absence of extinction, two causes are simultaneously responsible for the wavelength dependence of ellipticity profiles. First, the spatial distribution of the new population is very elongated along the bar because of new stars born in the gas flow along the bar that is narrower than the stellar bar. Then, secular evolution is responsible for progressively making it rounder. This explains the high ellipticity reached in the bar when the star-formation rate (SFR) is high ( $t<0.5$ Gyr for Run A) and, only in part, the subsequent decrease. Second, the luminosity ratio between the new and the initial populations is wavelength dependent, being higher in $B$ than in $K$ band. After the SFR maximum, the morphology becomes gradually dominated by the luminosity of the initial population that has a rounder spatial distribution. When dust extinction is taken into account, especially in the $B$ band, there is no longer a unique $e^{\max }$ because the real maximum is located in the dustiest region (e.g. $t=0.3$ Gyr in Fig. 8). A comparison between $B$ and $K$-band $\mathrm{E}_{1}$ measurements confirms that ellipticity is strongly dependent on the colour.

It is interesting to compare the maximum ellipticity with the bar strength $Q_{\mathrm{b}}$ defined as (Combes \& Sanders 1981):

$Q_{\mathrm{b}}=\max \left(\frac{F_{\theta}^{\max }(r)}{\left\langle F_{\mathrm{R}}(r)\right\rangle}\right)$

where $F_{\theta}^{\max }(r)$ is the maximum tangential force at radius $r$ and $\left\langle F_{\mathrm{R}}(r)\right\rangle$ is the average radial force from the axisymmetric component. Recently, this bar strength estimator has been also used in photometric studies (Buta \& Block 2001; Buta et al. 2005). Comparison of the evolution of the bar ellipticity with that of $Q_{\mathrm{b}}$ (cf. Fig. 10) clearly confirms that the bar axis ratio (or ellipticity) is not an estimator of the bar strength $Q_{\mathrm{b}}$. Indeed, bars with larger quadrupole moments and lower axis ratios can have the same $Q_{\mathrm{b}}$ as bars with smaller quadrupole moments and higher axis ratios. Moreover, the radius at which $Q(r)$ reaches its maximum, used to define $Q_{\mathrm{b}}$, is always located in the circumnuclear region. Thus, $Q(r)$ or $Q_{\mathrm{b}}$ is not useful for determining the bar radius. 
$\mathrm{T}=0.2(\mathrm{Gyr})$

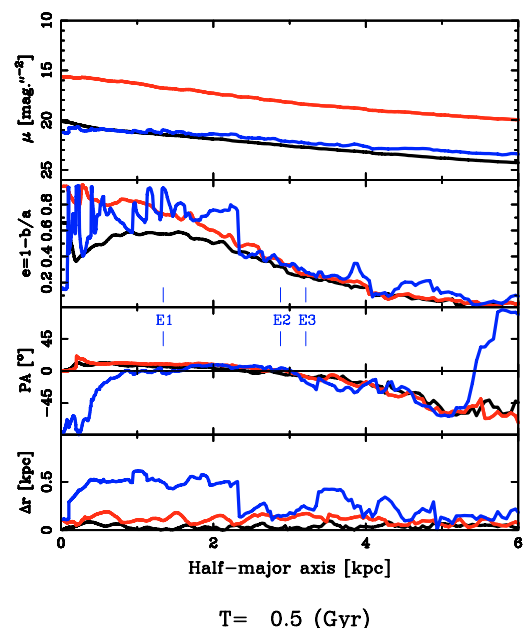

$\mathrm{T}=0.5$ (Gyr)

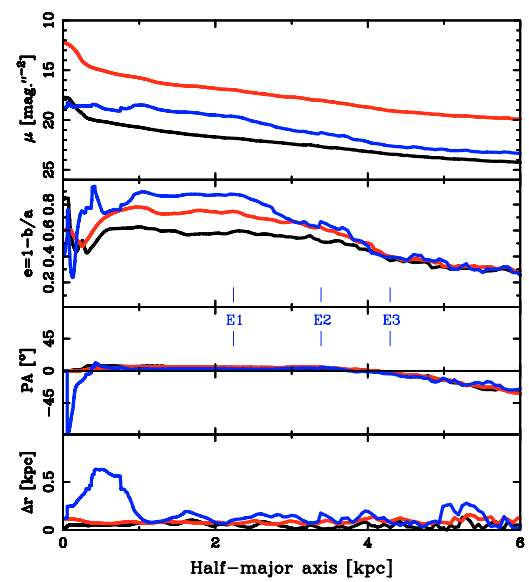

$\mathrm{T}=0.3(\mathrm{Gyr})$

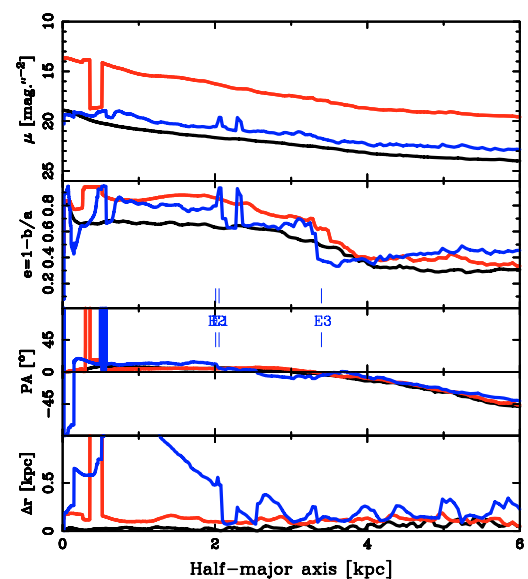

$\mathrm{T}=0.6(\mathrm{Gyr})$

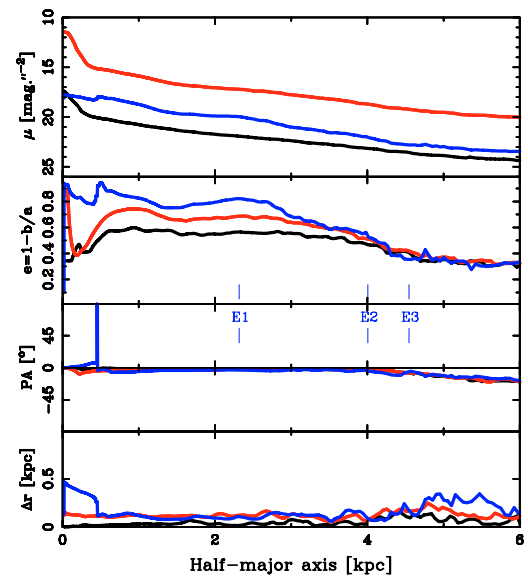

$\mathrm{T}=0.4(\mathrm{Gyr})$

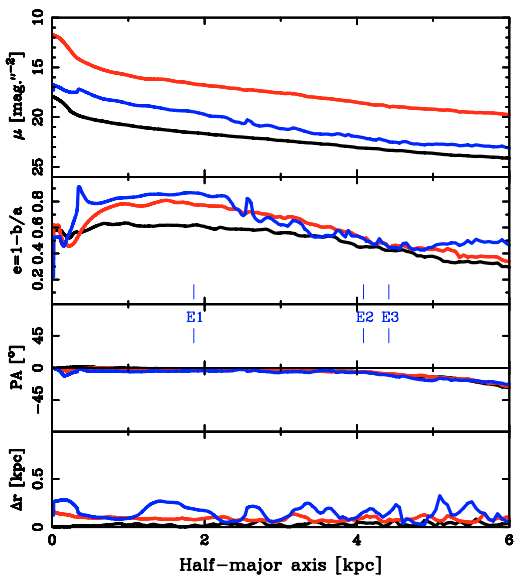

$\mathrm{T}=1.0(\mathrm{Gyr})$

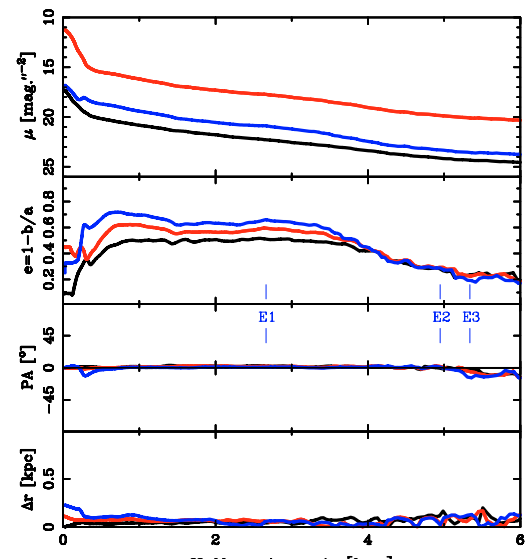

Fig. 8. Like Fig. 7 but for dusty images. Results for the mass density are the same than in Fig. 7 since they are not affected by extinction.

The distributions of $Q_{\mathrm{b}}$ for our simulations and for real galaxies (Buta et al. 2005) are very similar, except for $Q_{\mathrm{b}}<0.15$ (very weak bars). Bar morphology and properties, such as strength and ellipticity, produced in our simulations are thus representative of real galaxies in the local universe.

Due to the definition of $E_{2}$ and $E_{3}, P A$ variations are $\lesssim 10^{\circ}$ within the radial range defined by $E_{2}$ and $E_{3}$ criteria. This region of strong isophotal twist clearly marks the boundary of the bulk of the bar. Outside this transition region the surface photometry is dominated by the disc contribution and possibly by spiral arms; however, the size of this twisted region could be very time dependent, especially in the presence of spiral arms. This leads to variation from one snapshot to the next in $E_{2}$ and $E_{3}$ bar radii since the region could shrink or expand on a short timescale.

Fourier analysis was also done for each mock image. Radial profiles of the contrast computed with $B$ and $K$ images, as well as with the mass distribution, are displayed in Fig. 9 for Run A. In general, the contrast follows a well-defined curve, with a clear maximum once a bar is formed. However, during the most active star-formation phase, large fluctuations of the contrast, especially in the dust-free $B$ band, make it difficult to draw any radial profile. The contrast is also very sensitive to the extinction in the $B$ band, as opposed to the $K$ band. Interestingly, the contrast is always higher in the $B$ band than in the $K$ band. The lowest values are reached for the mass distribution. With another observational tool, this confirms the result found in Paper I that the bar shape (ellipticity or contrast) depends both on the wavelength and on the delay after the major star-formation phase.
However, it could be difficult or even impossible to define a maximum (e.g. $t=0.3$ or $t=0.4 \mathrm{Gyr}$ in Fig. 9). Moreover, the minimum could sometimes be negative in the case of very elongated and thin-light distribution, which is the case when star formation is active along the gas streamlines in the bar. Indeed, $I_{\text {ib }}$ takes negative values. This also explains why we failed to apply the $\mathrm{F}_{1}$ criterion to dust-free $B$ images. Sometimes, the contrast remains lower than 2 everywhere (as for the test image in Sect. 2.2), which makes it impossible to obtain $F_{1}$.

\subsection{Projection and resolution effects}

Because real galaxies are never exactly face-on, it can be asked how the bar radius determined is affected by projection effects when using the six criteria. We used one snapshot (Run A at $t=$ $0.77 \mathrm{Gyr}$ ) to study, on one hand, the effects of spatial resolution and, on the other, the effects of inclination. We used images in $K$ band.

When working with inclined galaxies, we also had to take the PA of the bar with respect to the line of node into account. Therefore we measured the radius of the bar using the six criteria for simulated galaxy images inclined from $15^{\circ}$ (nearly faceon) to $70^{\circ}$. For each inclination, we used 4 values for the bar position-angle $\left(\mathrm{PA}=0^{\circ}, 30^{\circ}, 60^{\circ}\right.$, and $\left.90^{\circ}\right)$.

For $i=15^{\circ}$, the measurements of bar radii are rather insensitive to the PA variation. For $i>15^{\circ}$ there are two regimes. For $\mathrm{PA}<60^{\circ}$, bar radii decrease with $\mathrm{PA}$, and this decrease is stronger for higher values of inclination. For PA $>60^{\circ}$, the trend 

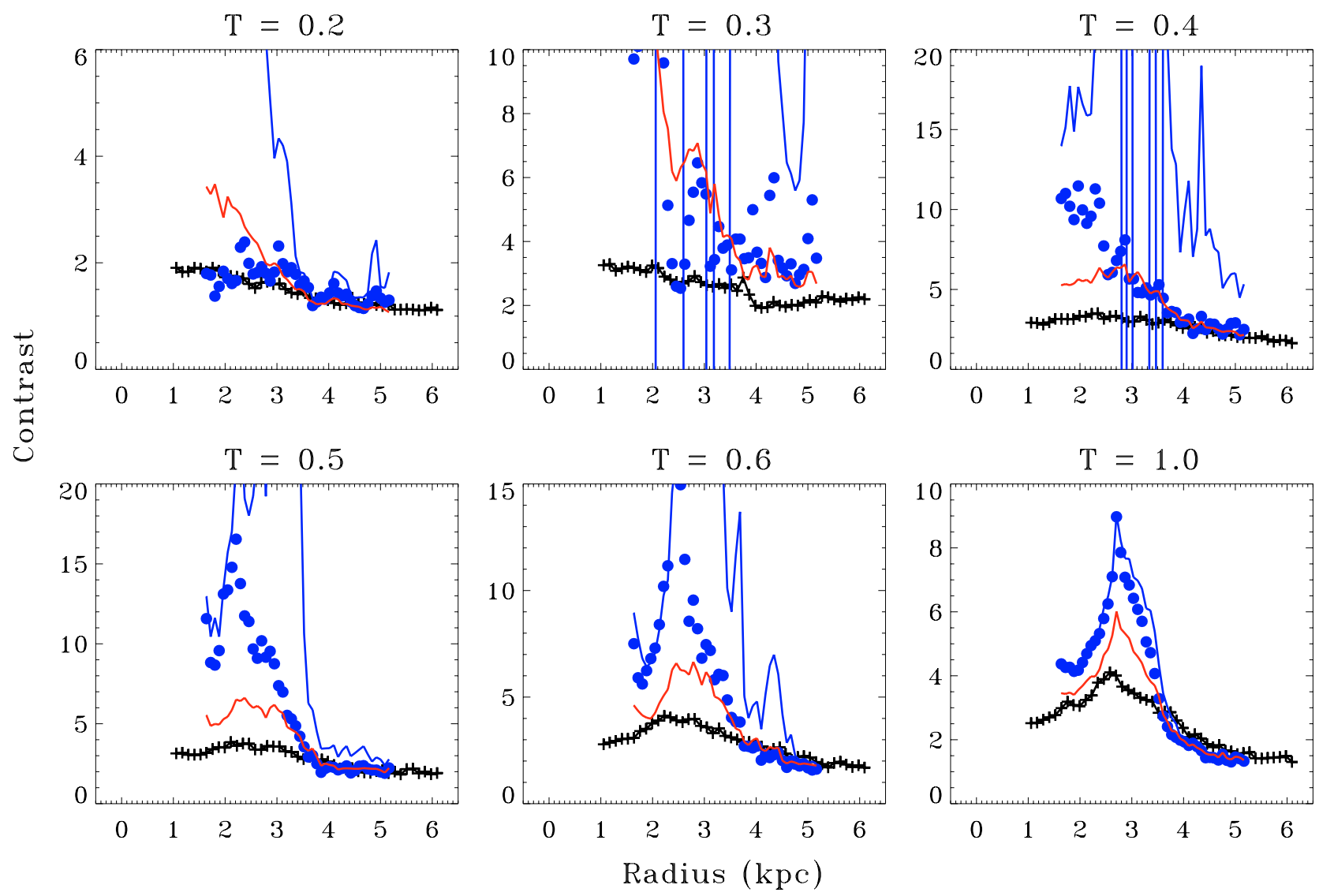

Fig. 9. Contrast profiles inside the central $6 \mathrm{kpc}$ of Run A, determined on the mass distributions (black crosses), $B$ images with extinction (blue full circles), dust-free $B$ images (blue line), and $K$ images (red line). Measurements inside the central kpc are not plotted since they are not reliable.

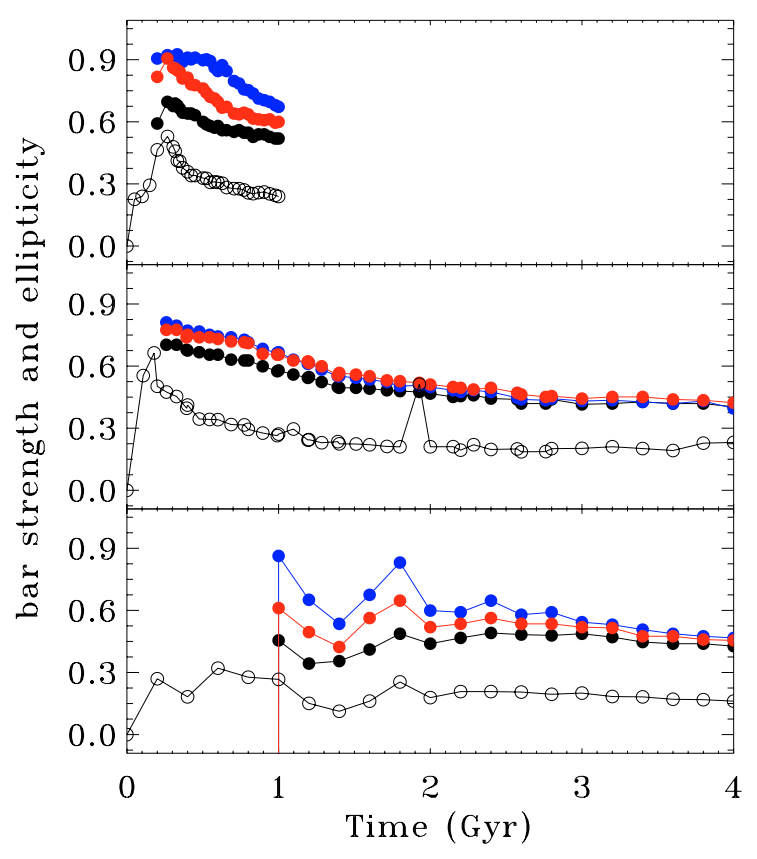

Fig. 10. Evolution of the bar strength $Q_{\mathrm{b}}$ (open circles) for Runs A (top), $\mathrm{B}$ (middle), and C (bottom). The maximum ellipticity of the bar is plotted as full circles for the mass density (black), $B$ images (blue points), and $K$ images (red points).

depends on the criterion. For example, bar radii determined with $F_{1}$ and $F_{2}$ decrease with PA, whereas they increase for $E_{2}$. With the $E_{1}$ criterion, the bar radius is almost constant in this range of $i$ and PA. For $\mathrm{E}_{3}$ and $\mathrm{F}_{3}$, bar radii depend on the degree of inclination. In addition for $i=70^{\circ} \mathrm{F}_{1}$ does not work, and for high values of $i$ and low values of PA, $\mathrm{E}_{1}$ gives a bar radius close to the values obtained with $E_{2}, F_{2}$, and $F_{3}$.

To give a rough estimate of the error made on the bar length using imperfectly deprojected images of inclined galaxies, we find that in all cases the difference for $i<30^{\circ}$ is less than $20 \%$ and the difference for $i<15^{\circ}$ is less than $10 \%$. For our images, with a bar radius at $5 \mathrm{kpc}, 10 \%$ represents less than 5 pixels.

Finally one could also wonder how the spatial resolution of the images affects the determination of the bar length. We then measured the bar radius using those six criteria in $K$-band images with lower resolution (two to five times lower resolution; the lowest resolution is then approximatively $0.6 \mathrm{kpc}$ per pixel). For a two-times lower resolution, there is no difference: variation lower than $2 \%$, which is lower than incertitudes due to human measurement. For lower resolution, bar-radius measurements with $\mathrm{E}_{2}, \mathrm{~F}_{1}$, and $\mathrm{F}_{2}$ have small variations $(\approx 5 \%)$, whereas with $\mathrm{E}_{1}, \mathrm{E}_{3}$, and $\mathrm{F}_{3}$ they vary more significantly $(10-15 \%)$. When resolution is decreased, the trends are that bar radius determined with $E_{2}$ decreases slightly, increases slightly with $F_{1}$ and $F_{2}$, increases with $\mathrm{E}_{3}$, and decreases with $\mathrm{F}_{3}$. The $\mathrm{E}_{1}$ criterion is more fluctuating, and bar radius is then smaller or longer, depending on the resolution. Due to these effects, $E_{1}, E_{2}$, and $F_{3}$ criteria could give close values for the bar radius at low resolution.

\subsection{Dust extinction effects}

Extinction effects in the bar region are important. For Run A at $t \approx 0.3 \mathrm{Gyr}$, less than $20 \%$ of the bar $B$-band luminosity escapes, 
increasing to $55 \%$ at $t \approx 1 \mathrm{Gyr}$. As shown in Paper I, $B$ isophotes are more disturbed by dust extinction than $K$ ones. Since our dust model is proportional to H I column densities, extinction decreases with time following the consumption of gas by star formation. A comparison of Figs. 7 and 8 shows that ellipticity profiles are completely different and very noisy when extinction is high, making bar-radius determination difficult. Differences in $B$-band ellipticity profile are still present at $t=1 \mathrm{Gyr}$ but mainly appear in the central kpc.

Regions with high SFR are located where gas density is the highest. In these regions, extinction is then very high since it is proportional to the gas column density. The regions that contain the youngest and bluest populations are also the most obscured. Since the gas is consumed during the evolution and since gas and stars have different kinematics, the gas density decreases secularly, enabling the escape of the blue light after several tens of Myr. However, the gas consumption occurs inhomogeneously, and at various rates, in the bar region.

All criteria are not affected by extinction in the same way. For instance, the $E_{3}$ criterion is in general rather insensitive to the extinction because the measurements are made in regions where the gas density is lower than in the innermost regions but, depending on the detailed spatial distribution of the gas, it could also lead to large errors. For Run $\mathrm{A}$, the $\mathrm{E}_{3}$ bar radius at $t=$ 0.6 Gyr differs by $1 \mathrm{kpc}$, whether extinction is present or not. It is also noteworthy that, although the $\mathrm{E}_{1}$ bar radii differ when extinction is added to the images, maximum ellipticity values do not significantly differ from the dust-free case. For Run B, the ellipticity and PA profiles in $B$ band are strongly affected by dust extinction during the 2 first Gyr. For most of these snapshots, we were not able to determine the bar radius using $E_{1}, E_{2}$, and $E_{3}$.

Paradoxically, the contrast profiles $C(r)$ are much more reliable when extinction is taken into account, since the very active regions along the gas streamlines are hidden. Fluctuations of $I_{\mathrm{ib}}$, the denominator in the definition of $C$, are thus strongly smoothed. The application of $F_{3}$ to dusty images leads to much more difficult measurements of bar radii, in particular in the $B$ band and during the first hundred Myr of evolution when the gas density is still high.

\section{Evolution of bar radius}

The evolution of the bar radius, determined using all six criteria, is plotted in Figs. 11 and 12. In the case of Runs A and B, for $t \lesssim 0.2 \mathrm{Gyr}$, the bar progressively grows but is still too weak to give a sharp signature in the surface-brightness distribution. Thus, each of the criteria fails to accurately define a bar radius during this growing phase. This is also the case for Run $C$ for $t \lesssim 1 \mathrm{Gyr}$, since the bar formation has a longer timescale due to the more massive initial bulge.

We hereafter discuss the results obtained for Run A and then look into details of the evolution on the longer term.

\subsection{Results with $E_{1}, E_{2}$, and $E_{3}$}

Criteria based on ellipse fitting give different estimations of the bar radius; $E_{1}$ gives generally lower values, while $E_{3}$ gives the highest estimation, as expected (cf. Sect. 2.1). However, $E_{1}$ and $\mathrm{E}_{2}$ in $B$ band give similar results during the active star-formation phase $(t \lesssim 0.6 \mathrm{Gyr}$ ) because of the steep ellipticity gradient due to the sharp bar boundary.

The bar radii for the mass density and $K$ images show the same trend as a function of time. They thus could be considered as similar on average, although results clearly show larger fluctuations for the mass density than for the $K$ images.

For the three criteria, the large fluctuations of bar radius from one snapshot to the next do not have a dynamical origin. This is only the consequence of our attempt to apply the criteria in the most objective way. Indeed, we systematically disregard barradius determination for previous snapshots. These fluctuations must be considered as an estimation of the error caused by using a criterion based on ellipse fitting. Since an ellipticity maximum is easier to determine than any other peculiar place on ellipticity or PA profiles, $E_{1}$ gives less noisy estimations than $E_{2}$ or $E_{3}$.

\subsection{Results with $F_{1}, F_{2}$, and $F_{3}$}

In comparison with the previous criteria, $F_{1}, F_{2}$, and $F_{3}$ give different radius estimations, evolution, and level of fluctuations. Indeed, the bar radius determined with $F_{1}$ and $F_{2}$ is roughly constant with a little dispersion for $t>0.5 \mathrm{Gyr}$, whereas it increases slightly with time and with a stronger dispersion for the other ellipse-based criteria. Bar radius determined with $\mathrm{F}_{3}$ first increases, then slightly decreases for $t>0.8 \mathrm{Gyr}$. For $\mathrm{F}_{1}$ and $\mathrm{F}_{2}$, the level of fluctuation from one snapshot to the next is lower than in the case of $E_{2}$ and $E_{3}$ because: 1) these criteria do not rely on human decision and 2) high frequencies in the image are smoothed out. However, $F_{3}$ is a bit noisier than $F_{1}$ or $F_{2}$ probably because this criterion also relies on human decision as do the $E_{2}$ and $E_{3}$ criteria. Moreover, $F_{1}$ and $F_{2}$ give similar results for the mass but differ on $K$-band images, $\mathrm{F}_{1}$ giving a longer bar than $\mathrm{F}_{2}$ by $\approx 1 \mathrm{kpc}$.

The $\mathrm{F}_{2}$ criterion deserves particular attention since it gives similar and reliable results for the mass, $B$, and $K$ images for $t>$ $0.5 \mathrm{Gyr}$, that is, $0.2 \mathrm{Gyr}$ after the peak of SFR. For $t<0.3 \mathrm{Gyr}$, when the bar is still growing, bar-radius estimation is unreliable. For $0.3<t<0.5 \mathrm{Gyr}$, when star formation is the most active, the bar radius is shorter in $B$ than in $K$, the highest value being obtained for the mass. However, the contrast $C$ profiles (Fig. 9) clearly show that these estimations are not reliable, apart from the mass density.

Let us recall that it was not possible to get valuable bar radii with $\mathrm{F}_{1}$ on $B$-band images because the contrast often remains above the threshold (fixed at 2 by Ohta et al.) until the end of the bar.

\subsection{Long-term evolution}

All the above analysis were applied to the other two runs that were performed until $t=7 \mathrm{Gyr}$. We reached roughly similar conclusions when we compared the results of the three runs. Such a comparison does make sense only on condition that the different evolution histories of each run are taken into account. The first Gyr of Run A is therefore comparable to almost the first $1.5 \mathrm{Gyr}$ of Run B and the first 3 Gyr of Run C.

The differences between Run $A$ and the two other runs mainly concern the criteria $E_{1}$ and $F_{1}$ to $F_{3}$. For Run $B$, the $F_{1}$ criterion can be measured from $B$-band images, and the results are similar to those in $K$ band. For Run $C$, the measurements using this criterion are possible and reliable for $t \gtrsim 2 \mathrm{Gyr}$ and give the same values as the $F_{2}$ criterion. Another difference concerns the $E_{1}$ criterion for Run C. Indeed, it gives approximatively the same values as the $\mathrm{E}_{2}$ criterion for $t \lesssim 3 \mathrm{Gyr}$. The $\mathrm{F}_{3}$ criterion gives a significantly greater bar radius than those obtained with $F_{1}$ and $F_{2}$. This criterion is very comparable to $\mathrm{E}_{2}$. 


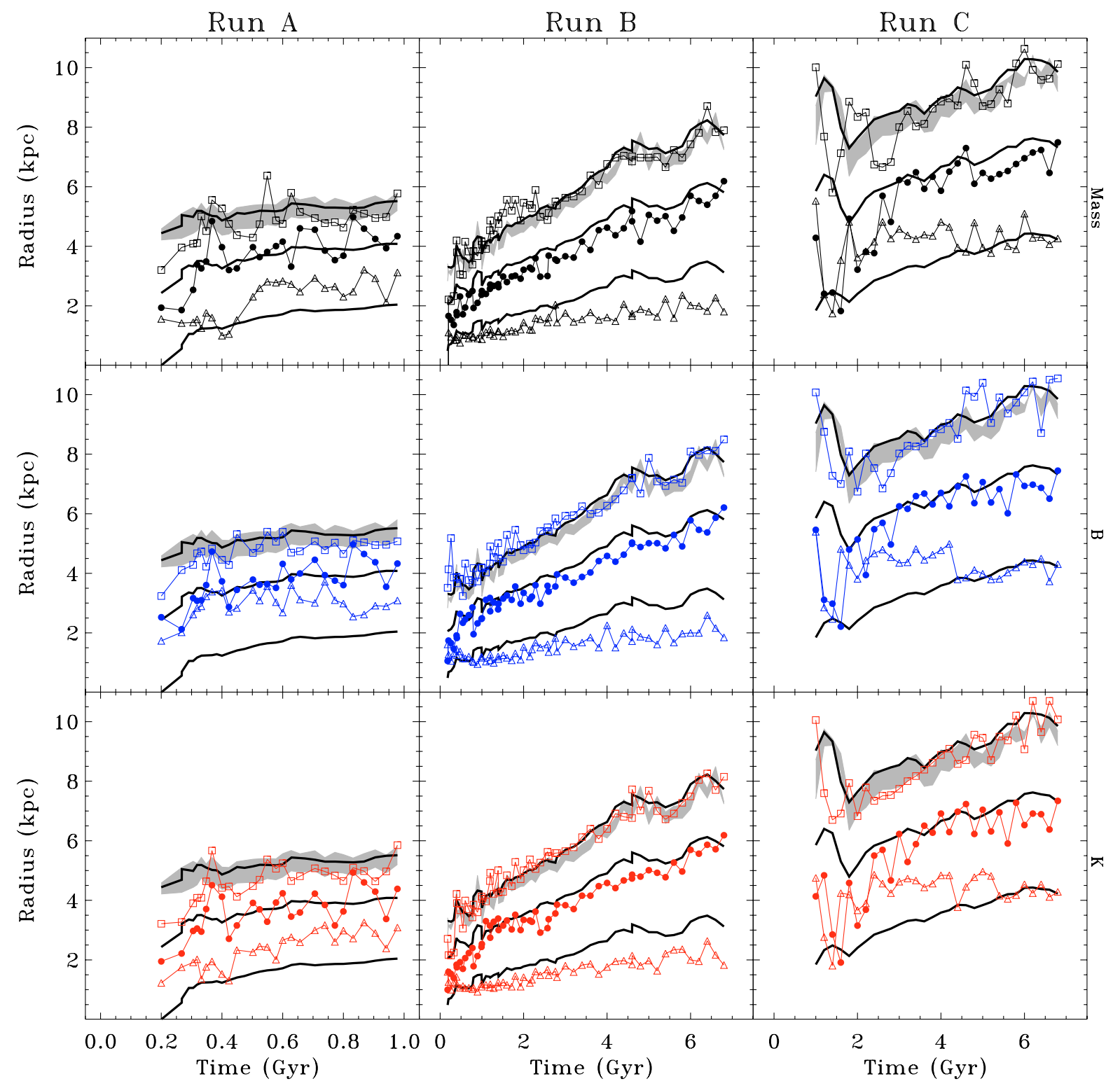

Fig. 11. Bar and resonance radii for Runs A, B, and C. For each panel, lines represent the ILR (shorter radius), UHR (intermediate radius), and CR (greater radius). The dimmed region shows the $L_{1,2}-L_{4,5}$ range. Bar radii determined with the maximum ellipticity criterion ( $\left.E_{1}\right)$ are plotted as open triangles, those determined with the minimum ellipticity criterion $\left(E_{3}\right)$ are plotted with open squares, and the new criterion $\left(E_{2}\right)$ is represented by full circles. Top panels: bar radii determined for the mass. Middle panels: $B$ dust-free images. Bottom panels: $K$ dust-free images.

On the other hand, for the criteria $E_{2}, E_{3}$, and $F_{1}$ to $F_{3}$, each gives similar results when we determine bar radius on the $B$ or $K$-band images or on mass distributions, taking the uncertainties of measurements into account.

\section{Discussion}

\subsection{Comparison with dynamical radii}

In Fig. 11 we also plot the radius of the CR $\left(R_{\mathrm{CR}}\right)$, ILR, and UHR resonances. In order to estimate the dynamical resonances radii, we computed the circular orbit frequency $\Omega$ and the radial epicyclic frequency $\kappa$ as (Pfenniger 1990):

$$
\begin{aligned}
& \Omega^{2}(r)=\left\langle\frac{1}{r} \frac{\partial \Phi}{\partial r}\right\rangle \\
& \kappa^{2}(r)=\left\langle\frac{\partial^{2} \Phi}{\partial x^{2}}+\frac{\partial^{2} \Phi}{\partial y^{2}}+2\left(\frac{1}{r} \frac{\partial \Phi}{\partial r}\right)^{2}\right\rangle
\end{aligned}
$$

where $\Phi$ is the gravitational potential and $\langle\cdots\rangle$ stands for an azimuthal average.

Strictly speaking, these frequencies predict the oscillation frequencies of the orbits only in the axisymmetric case. They do not indicate whether families of periodic orbits actually follow such oscillations when the bar growth breaks the axisymmetry. In fact, a proper orbital analysis is more appropriate. However, a number of previous orbital studies incline us to consider that the epicyclic approximation could lead to an acceptable estimation of the resonance locations.

Indeed, for the ILRs, Athanassoula (1992a) has computed the spatial extension of the $x_{2}$ and $x_{3}$ families associated with the existence of these resonances. Her comparison with ILR radii calculated by averaging the frequencies over the azimuthal angle shows that the error cannot exceed $10 \%$ in the worst cases. Since our $N$-body models are well inside the parameter space used by Athanassoula (1992a), we confidently use the linear approximation to estimate the radius of the outer ILR. 


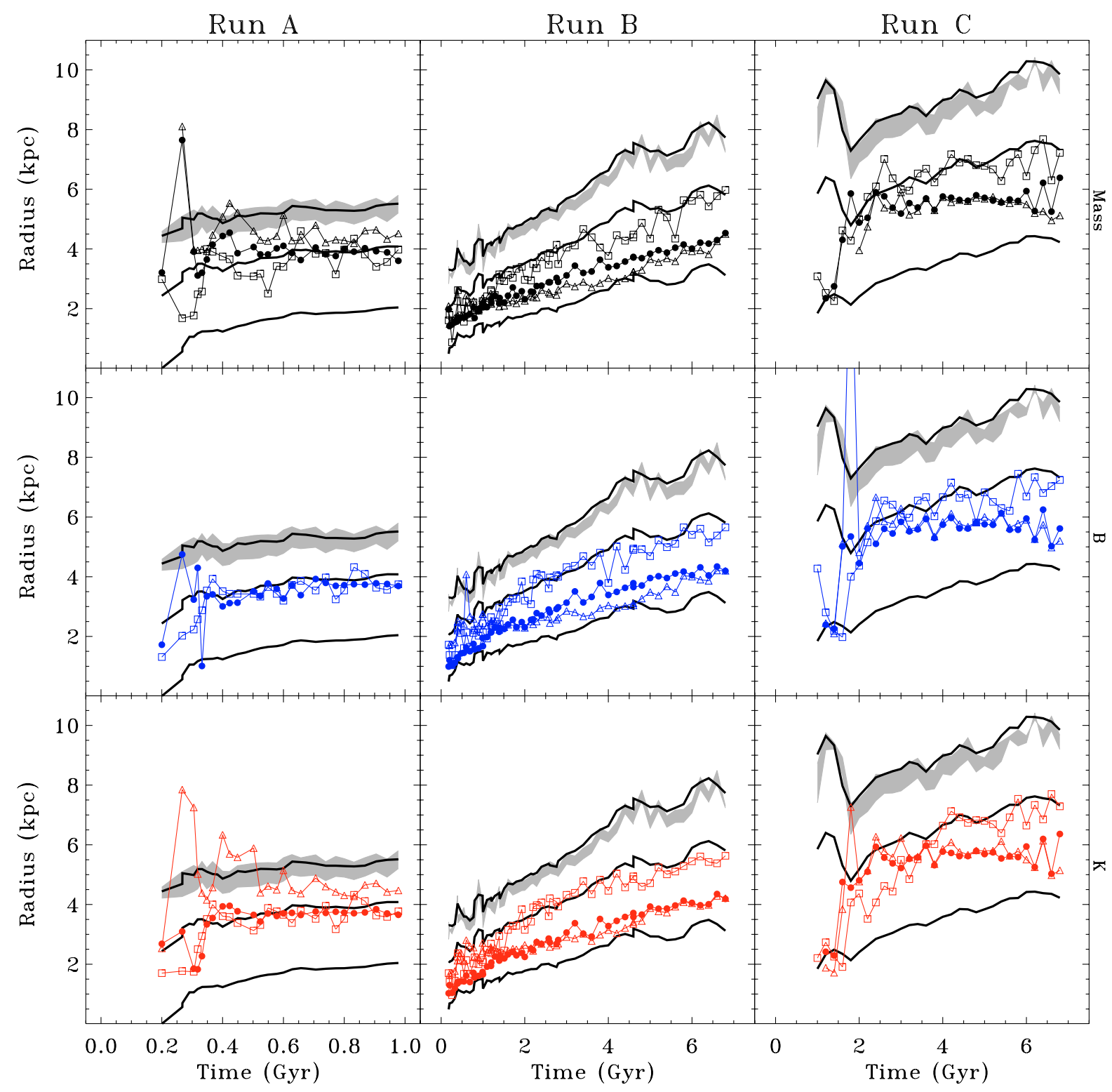

Fig. 12. Like Fig. 11 but for bar radii determined with the Ohta et al. criterion ( $F_{1}$, open triangles), Aguerri et al. criterion ( $F_{2}$, full circles), and $m=2$ phase criterion ( $\mathrm{F}_{3}$, open squares). Top left panel: measurements for the mass. Middle panels: $B$ dust-free images. Measurements for $t<0.4$ Gyr are not reliable for Run A (see text). Bottom panels: $K$ dust-free images.

To our knowledge, the differences between the real Lagrangian points location and the axisymmetric corotation radius have been less quantified in the past. In the case of barred galaxies, Lagrangian points are defined as the points of equilibrium between centrifugal and centripetal forces along the main axis of the bar perturbation. The radii of these points converge towards the corotation circle when the bar perturbation vanishes. Following Pfenniger (1990) and assuming a perfect bisymmetric barred potential, we call $L_{1,2}$ and $L_{4,5}$ the radii of the Lagrangian points along the major-axis and intermediate axis (minor-axis in a face-on projection), respectively, of the bar perturbation. In a strong bar potential, $L_{4,5}$ is slightly smaller than $L_{1,2}$. However, the amplitude of the difference between $L_{1,2}$ and $L_{4,5}$ should be roughly proportional to the bar strength.

In Fig. 13 we thus display the quantity $\Delta L=\left(L_{1,2}-L_{4,5}\right) / \bar{L}$, where $\bar{L}$ is the average between $L_{1,2}$ and $L_{4,5}$. The relative amplitude of the difference between $L_{1,2}$ and $L_{4,5}$ rarely exceeds $15 \%$ even in very strong bar phases, as for instance during the bar-formation phase in Run B. A standard value for a slowly evolving bar seems to be in the range 5 to $10 \%$. A comparison with the $Q_{\mathrm{b}}$ evolution clearly confirms this expected trend. When the bar gets stronger, the difference between $L_{1,2}$ and $L_{4,5}$ increases.

In the epicyclic approximation, corotation is the radius at which $\Omega\left(R_{\mathrm{CR}}\right)=\Omega_{\mathrm{b}}$, where $\Omega$ is the circular orbit frequency and $\Omega_{b}$ is the bar pattern speed. The value of $\Omega_{b}$ was accurately determined using the Tremaine-Weinberg method (Tremaine \& Weinberg 1984) since it has been shown that the method is very reliable on stellar velocity fields (Hernandez et al. 2005), i.e. the same values than those obtained using the phase of the bar. In Fig. 11 we compare the corotation radius with $L_{1,2}$ and $L_{4,5}$. As expected, the corotation radius lies between the Lagrangian points but is closer to $L_{1,2}$ than $L_{4,5}$. Thus, we can use the linear approximation to compute the corotation radius and $\Delta L$, as defined above, could be used as an estimator of the error made on the corotation location. 


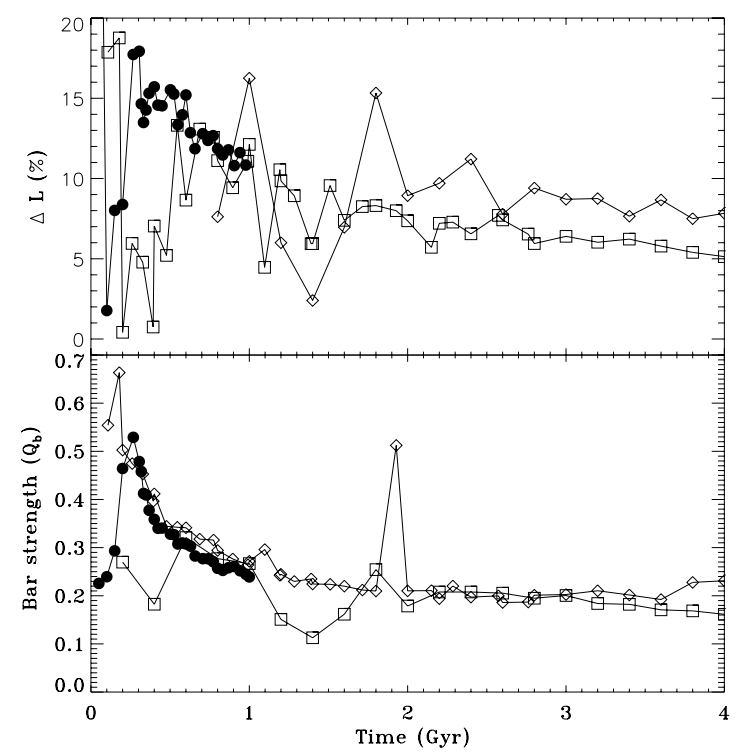

Fig. 13. Evolution of the relative difference between $L_{1,2}$ and $L_{4,5}$ (top) and bar strength $Q_{\mathrm{b}}$ (bottom) for Runs A (full circles), B (lozenges), and C (squares).

Keeping in mind a typical error of $10 \%$, as discussed above, we have determined the position of the radial ILR defined as the solution of $\Omega\left(R_{\mathrm{ILR}}\right)-\kappa\left(R_{\mathrm{ILR}}\right) / 2=\Omega_{\mathrm{b}}$ and UHR for which $\Omega\left(R_{\mathrm{UHR}}\right)-\kappa\left(R_{\mathrm{UHR}}\right) / 4=\Omega_{\mathrm{b}}$.

The last check was to compute the orbital frequencies $\kappa$ and $\Omega$ of a representative sample of particles. We applied a variant of the technique of Athanassoula (2003). We froze the potential at a given time in the simulation and then computed orbits in the inertial potential to determine the main frequencies using the technique of Carpintero \& Aguilar (1998). We used between 100000 and 200000 particles as initial conditions chosen at random. This leads to the determination of $\kappa$ and $\Omega$ for each particle which can be plotted in a $\kappa-\Omega$ diagram. Resonant families (e.g. ILR) and corotation are thus easily identifiable. We repeated this very CPU time-consuming computation ${ }^{1}$ for 4 snapshots in order to check the consistency with the linear approximation described above. We thus determined that the error on the corotation radius never exceeds $10 \%$.

The resonance locations are obviously independent of our photometric calibration and thus could be compared to our estimation of bar radii using various criteria. However, for all the reasons mentioned in Sect. 4 about $e^{\max }, \mathrm{E}_{1}$ cannot be, a priori, a good estimator of any dynamical radius (ILR, CR, etc.). This is clearly confirmed in Fig. 11. Also, $\mathrm{E}_{3}$ gives bar radii that are very close to the corotation. This strongly suggests that the ellipticity minimum at the end of the bar could be due to the signature of the corotation radius. However, if we define the corotation region as between $L_{1,2}$ and $L_{4,5}, \mathrm{E}_{3}$ gives a lower limit of the corotation.

The criteria $E_{2}, F_{2}$, and $F_{3}$ give bar radii that are rather well correlated with the UHR radius. However, $F_{2}$ is less correlated when SFR is high (for $t<0.5 \mathrm{Gyr}$ ), whereas $\mathrm{E}_{2}$ could give a higher error on the UHR position. One more difference appears between various simulations for the $\mathrm{F}_{2}$ criterion. For Run $\mathrm{C}$, it is correlated with the UHR radius for $t \lesssim 3.5 \mathrm{Gyr}$, i.e. for the duration comparable to Run A. On longer timescales, this is no longer the case. For Run B, the $F_{2}$ criterion never appears to be correlated with the UHR radius, and it gives values between the ILR and the UHR radius. Nevertheless, $\mathrm{E}_{2}$ and $\mathrm{F}_{3}$ criteria are still

${ }^{1}$ It takes roughly two weeks by snapshot on a 12-node cluster for 200000 orbits. correlated with the UHR radius on longer timescales, like the $E_{3}$ criterion with the corotation radius.

The fact that $E_{2}, F_{3}$, and in some cases $F_{2}$ criteria are good estimators of the UHR radius during the first Gyr can be understood in the framework of stellar orbits. Indeed, it has been shown (Contopoulos 1981a) that the UHR (or 4/1 resonance) is a gap where no family of periodic orbits can exist. Moreover, between the UHR and the corotation, most families of periodic orbits are unstable (Contopoulos 1981b) if the bar perturbation is stronger than $10 \%$ of the potential background (the stellar disc). Using 3D models, Skokos et al. (2002) also conclude that the most appropriate orbits to sustain a bar are those inside the UHR. Thus, between the UHR and the corotation, a bar is mostly sustained by semi-chaotic orbits (Wozniak \& Pfenniger 1999). The density response of these semi-chaotic orbits in the configuration space is rounder than the response density of orbits trapped around the major families of periodic orbits (i.e. $x_{1}$ and 3/1 resonant family). Thus the contrast as defined by Eq. (1) should strongly decrease after the UHR. This also explains why $E_{3}$ is a good tracer of the corotation radius.

However, for Run $C$, the $F_{2}$ criterion is no longer correlated with the UHR radius from $t \gtrsim 3.5 \mathrm{Gyr}$ and never appears to be correlated with the UHR radius for Run B. This means that this criterion is not a reliable estimator of the resonance location. The bar-interbar contrast indeed shows the pattern of the bar for all the snapshots of these three runs, but the decrease of the contrast at the end of the bar, which is the transition region between the bar and the disc, seems to be very sensitive to local conditions (such as star formation at the end of the bar, the spiral structure in the disc, and the thickness of the disc); therefore, these measurements depend on the type of bar and evolution. Then, even if the $F_{2}$ criterion is an automatic and reliable criterion of the bar radius, it defines a radius that is not always correlated with a resonance and then is not comparable from one to another galaxy.

\subsection{Fast and slow bars}

We plotted the corotation versus the two more accurate determinations of bar radius (cf. Fig. 14). The corotation radius was estimated using $\bar{L}=\left(L_{1,2}+L_{4,5}\right) / 2$ as in Sect. 6.1. The error is given by the difference between $L_{1,2}$ and $L_{4,5}$.

Using such a plot, Aguerri et al. (2003) have divided fast bars $\left(1<\mathcal{R}=R_{\mathrm{CR}} / R_{\mathrm{bar}}<1.4\right)$ from slow ones $(\mathcal{R}>1.4)$. For our simulations, especially for the long Runs B and C, $\mathcal{R}$ does not evolve very much with time except during the bar-formation phase. Once the bar is settled in, $\mathcal{R}$ remains rather constant.

It is noteworthy that the value of $\mathcal{R}$ is very sensitive to the definition of the bar radius. For the same simulation (either Run $\mathrm{B}$ or $\mathrm{C}$ ), $\mathrm{E}_{2}$ (or $\mathrm{F}_{3}$ ) leads to the conclusion that the bar is a rather slow rotator, while $E_{3}$ leads to the opposite conclusion. There is unfortunately no obvious theoretical definition of the "radius" or "length" of the bar. The only clean theoretical prediction is that bars cannot extend outside the corotation radius (Contopoulos 1980), whereas they could be limited by the disc scale length and thus could end near the ILR (Combes \& Elmegreen 1993). Moreover, when the ratio of the bar radius to the corotation radius is given (e.g. $1.2 \pm 0.2$, cf. Athanassoula $1992 \mathrm{~b}$ ), the bar radius is in fact defined as the half-major radius of a Ferrers ellipsoid that is not an observable radius, so that any comparison with observational bar radii could be difficult.

In the case of the measurement of $\mathcal{R}$, the use of $\mathrm{E}_{3}$ should obviously be avoided. Indeed, $E_{3}$ points out the corotation, leading to a ratio $\mathcal{R} \approx 1$. Thus, $\mathrm{E}_{2}$ and $\mathrm{F}_{3}$ criteria, being loosely correlated 


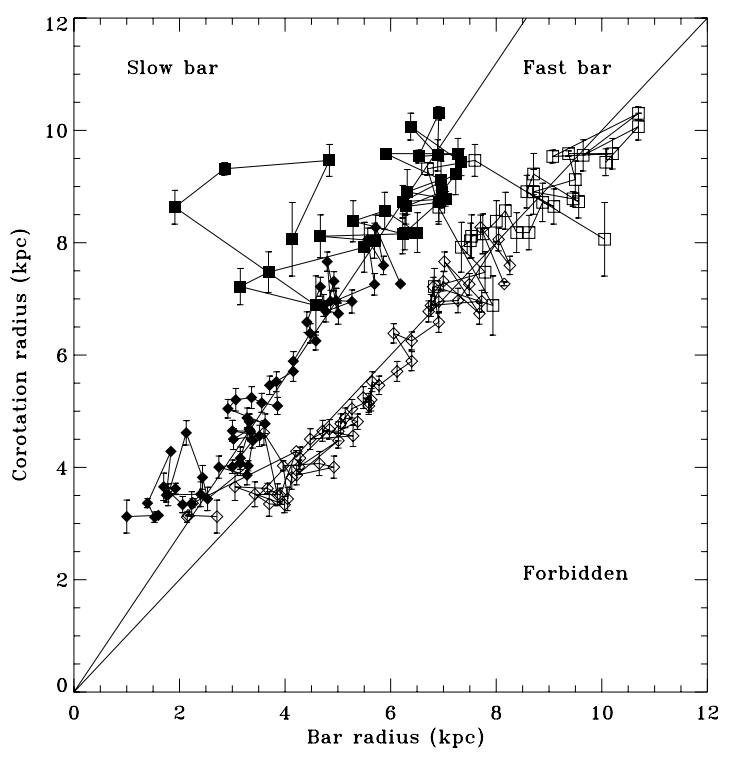

Fig. 14. Plot of corotation radius versus bar radius for Runs B (lozenges) and C (squares). The corotation radius was estimated using $\bar{L}$, and the error is given by the difference between $L_{1,2}$ and $L_{4,5}$. For the bar radius, we used criteria $E_{2}$ (full symbols) and $E_{3}$ (open symbols), determined on $K$-band images.

to a dynamical radius (UHR), could be useful in a first estimation of a bar radius. Defining the bar radius in such a way could be considered as a loose definition of the bar radius since the UHR does not limit the extension of the bar. We can thus imagine converting $R_{\mathrm{UHR}}$ into $R_{\mathrm{CR}}$, but the ratio $R_{\mathrm{CR}} / R_{\mathrm{UHR}}$ decreases from 1.5-1.8 during the short bar-formation phase to $\approx 1.3$ when the bar is settled in. Thus, the location of the corotation could only be retrieved within an error of the order of $10 \%$. Of course, the combination of the $\mathrm{E}_{2}$ criterion with $\mathrm{E}_{3}$ could lead to a better estimation of the corotation radius.

\subsection{Dark-halo effects}

The main effect of a live dark halo (except to flatten the rotation curve of the disc at large distance) is to permit exchanges of angular momentum with the stellar disc. The rate and amount of these exchanges depend on the velocity dispersion of both the disc and the halo, and on the relative halo mass (cf. Debattista \& Sellwood 2000; Athanassoula 2003; Valenzuela \& Klypin 2003). The stellar disc could lose between a few $\%$ and $40 \%$ of its angular momentum. Depending on the rate at which the stellar disc loses its angular momentum, the bar grows quite differently. The lack of dark halo in our simulations thus has mainly three consequences on our work:

1) The evolution timescale could be different from simulations with a dark halo, leading us not to draw any conclusion about the evolution of $\mathcal{R}$, for instance.

2) The shape and strength of a bar are also affected by the presence of the live halo (Athanassoula \& Misiriotis 2002). The central concentration of the halo is thus a key parameter. For instance, Athanassoula \& Misiriotis (2002) stress that more centrally concentrated halos lead to rectangularlike bars. However, the photometric and morphological properties (shape, ellipticity, $Q_{\mathrm{b}}$, etc.; cf. also Paper I) of our simulated bars are very representative of real stellar bars. For instance, we are able to reproduce a rectangular-like bar (Run B, Fig. 6).
3) The main results that could be affected by the absence of a dark halo in our simulations are those related to dynamical properties, i.e. the correlation of the position of resonances with bar-radii estimators (Sect. 6.1). This is a major concern since there is a debate on whether a dark halo increases $\mathcal{R}$ or not, leading to significantly smaller bars compared to corotation. Debattista \& Sellwood (2000) report high values of $\mathcal{R}$ for a number of their simulations. However, Debattista $\&$ Sellwood (2000) estimate bar radii using in part the $F_{3}$ criterion that is, for our simulations, quite correlated to UHR. This criterion gives rather short bars, since it is mainly influenced by the part of the bar sustained by $x_{1}$ orbits. This might explain the high $\mathcal{R}$ values in part. For the simulations by O’Neill \& Dubinski (2003) and Valenzuela \& Klypin (2003), $\mathcal{R}$ remains in the range $1.2-1.7$. Valenzuela \& Klypin (2003) mainly use a criterion based on surface density (not tested in the present paper), which seems to give similar results than $F_{3}$, whereas O'Neill \& Dubinski (2003) use an average of three methods (including $\mathrm{E}_{2}$ and $\mathrm{F}_{3}$ ).

Our results should thus be confirmed with simulations that include a live dark halo. However, the presence of a dissipative component that can also exchange angular momentum and mass with the collisionless components through gravitational interaction, star formation, and feedback processes must have some influence on the global angular momentum exchanges. Therefore, realistic models should now include both dark matter and a dissipative component. This will be published elsewhere.

\section{Conclusions}

We have used $N$-body simulations, including stars, gas, and star formation, that were photometrically calibrated in $B$ and $K$ wavebands to compare the bar lengths determined by six observational criteria (five commonly applied to observations or simulations and a sixth introduced in this paper). These criteria are based on ellipse fitting $\left(E_{1}\right.$ to $\left.E_{3}\right)$ and Fourier analysis $\left(F_{1}\right.$ to $\left.F_{3}\right)$ of the surface brightness (or mass surface density). The bar-length estimates were also compared to the location of resonances.

We have obtained the following main results for each criterion in turn:

- $E_{1}$ (Wozniak \& Pierce 1991), the radius of the maximum ellipticity, gives the shortest bar lengths. It clearly underestimates the real bar radius. Moreover, it is not linked to any dynamical characteristics of a bar (e.g. resonance).

- $E_{2}$ is measured at the end of the PA plateau. It can be used to approximately locate the UHR.

- $E_{3}$, the radius of the minimum of ellipticity (Wozniak et al. 1995), gives the greatest bar lengths. In general, it is less sensitive to dust absorption than other criteria. This is the only criterion, amongst the six criteria tested, that is correlated with the corotation.

- $F_{1}$ (Ohta et al. 1990) is not always defined, since the barinterbar brightness contrast $(C)$ could remain under 2 even for strong bars.

- $\mathrm{F}_{2}$ (Aguerri et al. 2000), also based on $C$, is the least noisy estimator. However it gives contradictory results for our simulations.

- $\mathrm{F}_{3}$, widely used to determine the bar length in $N$-body simulations (e.g. Debattista \& Sellwood 2000), gives bar lengths comparable to $\mathrm{E}_{2}$ results. Like $\mathrm{E}_{2}$, it gives quite a good approximation of the UHR location. 
In general, for projection angles $i$ between 15 and $70^{\circ}$, the bar length is underestimated with respect to its face-on value, apart from using $\mathrm{E}_{1}$. However, for $i<30^{\circ}$, errors remain below $20 \%$. Measurements also depend on the resolution but errors never exceed $15 \%$ when resolution is downgraded by a factor 5 .

We moreover confirm that ellipticity profiles of $B$ and $K$-band images can be very different. They also differ from ellipticity profiles of the mass density. This leads to a clear dependence of the maximum ellipticity on the waveband. However the radius of the maximum determined by $E_{1}$ is less affected. Within a typical error of $10 \%$, bar lengths determined with other criteria are not colour dependent, in the absence of dust. The situation is different when dust absorption is taken into account. Criteria giving the smallest bar-length estimate are much more affected than others. This is the case of $E_{1}$ in particular.

For each simulation, the maximum ellipticity decreases with time, as do the bar strengths $Q_{\mathrm{b}}$, but not at the same rate. The use of the maximum ellipticity to estimate the bar strength could thus lead to severe errors, especially in a comparison of one galaxy to another.

Accurate determination of the bar length is crucial in several observational or theoretical analyses. For instance, the ratio $\mathcal{R}=R_{\mathrm{CR}} / R_{\text {bar }}$ could increase from 1 (fast bar) to 1.4 (slow bar) just by using $E_{2}$ or $F_{3}$ instead of $E_{3}$. However, being correlated to the corotation radius, $E_{3}$ cannot be used to define the end of a bar. Using $E_{2}$ or $F_{3}$ to define the end of the bar has physical implications since these criteria point out the UHR. Indeed, inside the UHR, the bar is mainly sustained by $x_{1}$ or other families of orbits elongated along the bar major-axis. However, there is clear evidence that what is commonly called a bar extends a bit outside the UHR because the region between UHR and CR is populated by higher resonant families of orbits, as well as semichaotic and chaotic orbits that contribute to the shape (sometime rectangular-like) of the bar. The definition of the bar length (hence the criterion used) should thus depend on the application.

Acknowledgements. We are grateful to Luis Aguilar and an anonymous referee for comments and suggestions that have helped to strengthen the conclusions of this paper. We would also like to thank Luis Aguilar for providing his code to compute orbital frequencies. Our computations were partly performed on the Fujitsu NEC SX-5 hosted by IDRIS/CNRS and the CRAL 18-node cluster of PC funded by the INSU/CNRS (ATIP \# 2JE014 and Programme National Galaxie). L.M.D. acknowledges support from a grant from the Universidad Nacional Autónoma de México (UNAM) for part of this work.

\section{References}

Aguerri, J. A. L., Munoz-Tunon, C., Varela, A. M., \& Prieto, M. 2000, A\&A, 361, 841 Aguerri, J. A. L., Debattista, V. P., \& Corsini, E. M. 2003, MNRAS, 338, 465 Athanassoula, E. 1992a, MNRAS, 259, 328

Athanassoula, E. 1992b, MNRAS, 259, 345

Athanassoula, E. 2003, MNRAS, 341, 1179

Athanassoula, E., Morin, S., Wozniak, H., et al. 1990, MNRAS, 245, 130 Athanassoula, E., \& Misiriotis, A. 2002, MNRAS, 330, 35

Bruzual, G., \& Charlot, S. 1993, ApJ, 405, 538

Buta, R., \& Block, D. L. 2001, ApJ, 550, 243

Buta, R., \& Combes, F. 1996, Fund. Cosmic Phys., 17, 95

Buta, R., Vasylyev, S., Salo, H., \& Laurikainen, E. 2005, AJ, 130, 506 Carpintero, D. D., \& Aguilar, L. A. 1998, MNRAS, 298, 1

Chapelon, S., Contini, T., \& Davoust, E. 1999, A\&A, 345, 81

Combes, F., \& Sanders, R. H. 1981, A\&A, 96, 164

Combes, F., \& Elmegreen, E. E. 1993, A\&A, 271, 391

Contopoulos, G. 1980, A\&A, 81, 198

Contopoulos, G. 1981a, CMDA, 24, 355

Contopoulos, G. 1981b, A\&A, 102, 265

Debattista, V. P., \& Sellwood, J. A. 2000, ApJ, 543, 704

Erwin, P., \& Sparke, L. S. 2003, ApJS, 146, 299

Friedli, D., \& Benz, W. 1993, A\&A, 268, 65

Friedli, D., \& Benz, W. 1995, A\&A, 301, 649

Heller, C., Shlosman, I., \& Englmaier, P. 2001, ApJ, 553, 661

Hernandez, O., Wozniak, H., Carignan, C., et al. 2005, ApJ, in press

Jogee, S., Barazza, F. B., Rix, H.-W., et al. 2004, ApJ, 615, L105

Jungwiert, B., Combes, F., \& Axon, D. J. 1997, A\&AS, 125, 479

Kent, S. M. 1990, AJ, 100, 377

Knapen, J. H., Pérez-Ramírez, D., \& Laine, S. 2002, MNRAS, 337, 808

Kormendy, J., \& Kennicutt, R. C. 2004, ARA\&A, 42, 603

Laine, S., Shlosman, I., Knapen, J. H., \& Peletier, R. F. 2002, ApJ, 567, 97 Maeder, A. 1992, A\&A, 264, 105

Martin, P. 1995, AJ, 109, 2428

Martinet, L., \& Friedli, D. 1997, A\&A, 323, 363

Michel-Dansac, L., \& Wozniak, H. 2004, A\&A, 421, 863 (Paper I)

Miyamoto, M., \& Nagai, R. 1975, PASJ, 27, 533

Monaghan, J. J. 1992, ARA\&A, 30, 543

Ohta, K., Hamabe, M., \& Wakamatsu, K. 1990, ApJ, 357, 71

O'Neill, J. K., \& Dubinski, J. 2003, MNRAS, 346, 251

Patsis, P. A., Skokos, C., \& Athanassoula, E. 2002, MNRAS, 337, 578 Pfenniger, D. 1990, A\&A, 230, 55

Pfenniger, D., \& Friedli, D. 1991, A\&A, 252, 75

Pfenniger, D., \& Friedli, D. 1993, A\&A, 270, 561

Rautiainen, P., \& Salo, H. 1999, A\&A, 348, 737

Regan, M. W., \& Elmegreen, D. M. 1997, AJ, 114, 965

Regan, M. W., \& Teuben, P. J. 2004, ApJ, 600, 595

Sanders, R. H., \& Tubbs, A. D. 1980, ApJ, 235, 803

Sellwood, J. A., \& Wilkinson, A. 1993, Rep. Prog. Phys., 56, 173

Sparke, L. S., \& Sellwood, J. A. 1987, MNRAS, 225, 653

Skokos, C., Patsis, P. A., \& Athanassoula, E. 2002, MNRAS, 333, 861

Tremaine, S., \& Weinberg, M. D. 1984, ApJ, 282, L5

Valenzuela, O., \& Klypin, A. 2003, MNRAS, 345, 406

Wozniak, H., Friedli, D., Martinet, L., et al. 1995, A\&AS, 111, 115

Wozniak, H., \& Pfenniger, D. 1999, CDMA, 73, 149

Wozniak, H., \& Pierce, M. J. 1991, A\&AS, 88, 325 